Article

\title{
Dark Gravitational Field on Riemannian and Sasaki Spacetime
}

\author{
Panayiotis Stavrinos $1, * \mathbb{D}$ and Christos Savvopoulos ${ }^{2}$ \\ 1 Department of Mathematics, National and Kapodistrian University of Athens, 15784 Athens, Greece \\ 2 Department of Physics, National and Kapodistrian University of Athens, 15784 Athens, Greece; \\ sph1400190@uoa.gr \\ * Correspondence: pstavrin@math.uoa.gr; Tel.: +30-210-727-6416
}

Received: 8 July 2020; Accepted: 27 August 2020; Published: 28 August 2020

\begin{abstract}
The aim of this paper is to provide the geometrical structure of a gravitational field that includes the addition of dark matter in the framework of a Riemannian and a Riemann-Sasaki spacetime. By means of the classical Riemannian geometric methods we arrive at modified geodesic equations, tidal forces, and Einstein and Raychaudhuri equations to account for extra dark gravity. We further examine an application of this approach in cosmology. Moreover, a possible extension of this model on the tangent bundle is studied in order to examine the behavior of dark matter in a unified geometric model of gravity with more degrees of freedom. Particular emphasis shall be laid on the problem of the geodesic motion under the influence of dark matter.
\end{abstract}

Keywords: riemannian geometry; Sasaki metric; dark matter; geodesics; deviation of geodesics; Einstein equations; Raychaudhuri equations

\section{Introduction}

Recent advances in the theoretical and observational field of cosmology have shown the significance of the existence of dark matter and dark energy [1-25]. There is considerable evidence that most of the mass in the universe is neither in the luminous matter in galaxies, nor in the radiation detected so far. Mass can be detected by its gravitational influence even if it cannot be seen directly [26]. Evidence for the existence of dark matter comes for example from the study of gravitational lensing, cosmic microwave background radiation $(\mathrm{CMB})$ or the rotational curves of spiral galaxies $[27,28]$.

According to observational results, dark matter plays a dominant role in the field of evolution and acceleration of the universe. There are many pieces of evidence that the visible matter and detectable radiation comprise only a small fraction of the mass in the universe, perhaps as little as a few percent [26]. Therefore the study of dark matter is central for cosmology. A geometric model for gravity with dark matter based on a spacetime manifold endowed with a Riemannian metric is necessary, because of the existence of contributions to gravity, curvature, tidal forces etc. due to dark matter whose gravitational interaction could be described using a Riemmanian geometric framework similar to that of ordinary matter as shall be assumed throughout this work.

These contributions can be observed in the velocities of nearby trajectories (geodesics) of particles (or clusters thereof, e.g., planets), in the orbital motions of member galaxies in galaxy clusters or in the relative change of velocity of the deviation vector which shall now be altered to account for the existence of extra "dark" gravity. The above mentioned combined with observational data give rise to a question regarding whether or not one accepts the assumption that test bodies move on geodesics of a given inertial metric of the base spacetime [29,30]; in other words, whether or not the geodesics remain the same during the evolution of the universe with the addition of dark matter. 
In a more general geometric framework, the tangent bundle of a spacetime manifold, can extend the limits of a "unified" gravitational field in more than four dimensions. The development of the metric geometry of tangent bundles first began with the introduction of the natural Sasaki metric, in the fundamental paper of Sasaki published in 1958 [31], which we shall assume for a further geometric model with dark matter. This choice proves crucial as the tangent bundle allows for a gravitational field with more degrees of freedom. Dark matter could cause extra gravitational influence on all scales, which can be illustrated well in extra dimensions [32-36]. A geometric frame that potentially materializes this concept is, therefore, that of Sasaki, where the underlying metric structure is Riemannian.

This work is organized as follows: in Section 2, we examine the gravitational field with the addition of extra dark gravity in a Riemannian spacetime setting. In particular, in Section 2.1 we provide the geodesic equations and their deviation, in Section 2.2 we study the Einstein equations and in Section 2.3 the Raychaudhuri equation. In addition, in Section 2.4 we derive the Friedmann equations and the continuity equation as an application using the $F(R)$ gravity model for dark matter. In Section 3, we extend our study of the dark and ordinary gravitational field on the tangent bundle of a Sasaki spacetime. In Section 3.1 we derive the equations of geodesic deviation on the Sasaki tangent bundle, and in Section 3.2 we provide the foundations for an extension of dark gravity on the tangent bundle. Finally, in Section 4 we summarize the results of this work and in Appendix A we present some further geometric results.

\section{Dark Gravity in the Riemannian Spacetime}

Let us consider a (pseudo-)Riemannian 4-dimensional spacetime $M$ containing both matter and dark matter equipped with a metric $g$. For the purposes of our study, an additive relation for the metric tensor is assumed, such that the contributions to the metric of ordinary and dark matter can be viewed separately.

Let

$$
d s^{2}=g_{i j}(x) d x^{i} d x^{j}
$$

be the metric of this 4-dimensional spacetime, where we assume that the unified metric

$$
g_{i j}(x)=g_{i j}^{(O)}(x)+g^{(D)}{ }_{i j}(x)
$$

where $g^{(O)}{ }_{i j}(x)$ is the sectoral metric of ordinary matter and $g^{(D)}{ }_{i j}(x)$ that of dark matter ${ }^{1}$.

Due to the geometry of the space that has been chosen, we have to use the unified metric tensor, $g_{i j}$, for such operations as raising, lowering and contracting indices of tensors. Since, in our study, we deal with tensors related to ordinary or dark matter's spacetime, we shall attempt to relate such concepts within the framework of the unified geometric space. From a physical perspective, a unified framework of gravity which includes the gravitational interaction of ordinary and dark matter is necessary in order to describe the gravitational effects of the large scale universe structures (e.g., to explain the rotation of galaxies or the motions of clusters).

\subsection{Geodesics and Tidal Forces}

First, we find that the Chistoffel symbols of first kind are:

$$
\Gamma_{i j k}=\Gamma_{i j k}^{(O)}+\Gamma_{i j k}^{(D)}
$$

1 For all three metric tensors a metric signature $(-,+,+,+)$ shall be assumed in their respective space. 
where,

$$
\begin{aligned}
\Gamma^{(O)}{ }_{i j k} & =\frac{1}{2}\left(\frac{\partial g^{(O)}{ }_{i j}}{\partial x^{k}}+\frac{\partial g^{(O)}{ }_{i k}}{\partial x^{j}}-\frac{\partial g^{(O)}{ }_{j k}}{\partial x^{i}}\right) \\
\Gamma^{(D)}{ }_{i j k} & =\frac{1}{2}\left(\frac{\partial g^{(D)}{ }_{i j}}{\partial x^{k}}+\frac{\partial g^{(D)}{ }_{i k}}{\partial x^{j}}-\frac{\partial g^{(D)}{ }_{j k}}{\partial x^{i}}\right)
\end{aligned}
$$

are the Christoffel symbols of first kind of a space occupied exclusively by ordinary or dark matter, respectively ${ }^{2}$.

The geodesics will then be given by the well-known equation, which needs to be modified to account for the existence of dark matter as

$$
\frac{d^{2} x^{i}}{d t^{2}}+\left(\Gamma_{j k}^{(O) i}+\Gamma_{j k}^{(D) i}+\gamma_{j k}^{i}\right) \frac{d x^{j}}{d t} \frac{d x^{k}}{d t}=0
$$

since the Christoffel symbols of second kind shall have the following form:

$$
\gamma_{j k}^{i}=\Gamma_{j k}^{(O) i}+\Gamma_{j k}^{(D) i}+\gamma_{j k}^{i}
$$

where $t$ is an affine parameter and

$$
\begin{gathered}
\Gamma_{j k}^{(O) i}=\frac{1}{2} g^{(O) i l}\left(\frac{\partial g^{(O)} l j}{\partial x^{k}}+\frac{\partial g^{(O)} l k}{\partial x^{j}}-\frac{\partial g^{(O)} j k}{\partial x^{l}}\right) \\
\Gamma_{j k}^{(D) i}=\frac{1}{2} g^{(D) i l}\left(\frac{\partial g^{(D)} l j}{\partial x^{k}}+\frac{\partial g^{(D)} l k}{\partial x^{j}}-\frac{\partial g^{(D)}{ }_{j k}}{\partial x^{l}}\right)
\end{gathered}
$$

are the Christoffel symbols of ordinary and dark matter's metric contribution, respectively, and

$$
\gamma_{j k}^{i}=\gamma_{j k}^{i}-\left(\Gamma_{j k}^{(O) i}+\Gamma_{j k}^{(D) i}\right)
$$

is the interaction part of the Christoffel symbols since it could be shown that $\gamma_{j k}^{i}$ represents the interaction between the ordinary and dark matter gravitational potentials and their respective intensities ${ }^{3}$.

In view of relation (6), one can see that for a test particle $m_{i}$ moving along geodesics in the spacetime of our model the following relation holds:

$$
m_{i}\left(\frac{d^{2} x^{i}}{d t^{2}}+\Gamma^{(O) i}{ }_{j k} \frac{d x^{j}}{d t} \frac{d x^{k}}{d t}\right)=-m_{i}\left(\Gamma_{j k}^{(D) i}+\gamma_{j k}^{i}\right) \frac{d x^{j}}{d t} \frac{d x^{k}}{d t}=F_{(D)}^{i}
$$

Seemingly, the left-hand side of Equation (11) does not give a geodesic in the Riemannian sense since the right-hand side does not vanish unless we consider dark matter to be absent. The dark gravitational field and its interaction with ordinary matter influences the geodesics in a dominant way and give rise to a dark pseudo-force field $F_{(D)}^{i}$ which can be interpreted as a gravitational source for inertial force fields of interaction by an observer who does not take into account the existence of dark matter in his considerations. The addition of dark matter also influences the curvature tensor of the unified space, $R_{b c d}^{a}$, which assumes the form shown in the following relation:

2 One must be careful that $\Gamma^{(O)}{ }_{i j k}$ and $\Gamma^{(D)}{ }_{i j k}$ do not function as Christoffel symbols for the unified space $(M, g)$.

3 These $\gamma_{j k}^{i}$ symbols could be explicitly calculated using [37]. 


$$
R_{b c d}^{a}=R_{b c d}^{(O) a}+R_{b c d}^{(D) a}+r_{b c d}^{a}
$$

where,

$$
R_{b c d}^{(O) a}=\frac{\partial \Gamma^{(O) a} b d}{\partial x^{c}}-\frac{\partial \Gamma^{(O) a} b c}{\partial x^{d}}+\Gamma_{c e}^{(O) a} \Gamma_{b d}^{(O) e}-\Gamma_{d e}^{(O) a} \Gamma_{b c}^{(O) e}
$$

is ordinary matter's Riemann tensor,

$$
R_{b c d}^{(D) a}=\frac{\partial \Gamma^{(D) a} b d}{\partial x^{c}}-\frac{\partial \Gamma^{(D) a} b c}{\partial x^{d}}+\Gamma_{c e}^{(D) a} \Gamma_{b d}^{(D) e}-\Gamma^{(D) a}{ }_{d e} \Gamma^{(D) e} b c
$$

is dark matter's curvature tensor and,

$$
\begin{aligned}
r_{b c d}^{a}=\frac{\partial \gamma_{b d}^{a}}{\partial x^{c}}- & \frac{\partial \gamma_{b c}^{a}}{\partial x^{d}}+\Gamma_{e[c}^{(O) a} \Gamma_{d] b}^{(D) e}+\Gamma_{e[c}^{(D) a} \Gamma_{d] b}^{(O) e}+\Gamma_{e[c}^{(O) a} \gamma_{d] b}^{e} \\
& +\Gamma_{e[c}^{(D) a} \gamma_{d] b}^{e}+\gamma_{e[c}^{a} \Gamma_{d] b}^{(O) e}+\gamma_{e[c}^{a} \Gamma_{d] b}^{(D) e}+\gamma_{e[c}^{a} \gamma_{d] b}^{e}
\end{aligned}
$$

is the interaction part of the Riemann tensor ${ }^{4,5}$.

Similarly, the equation of geodesic deviation needs to be modified, as well. It will then take the following form:

$$
\delta_{t}\left(\delta_{t} W^{a}\right)=-\left(R_{b c d}^{(O) a}+R_{b c d}^{(D) a}+r_{b c d}^{a}\right) V^{b} W^{c} V^{d}
$$

where,

$$
\delta_{t} W^{a}=\frac{\partial W^{a}}{\partial t}+\left(\Gamma_{b c}^{(O) a}+\Gamma_{b c}^{(D) a}+\gamma_{b c}^{a}\right) W^{b} V^{c}
$$

$V^{i}=\partial_{t} x^{i}, W^{i}=\partial_{s} x^{i}$ and $x_{s}^{i}(t)$ is a two-parameter geodesic family with $t$ being the affine parameter and $s$ the selector parameter [26].

In view of relations (12) and (16), the extra terms of the product of the Christoffel symbols of ordinary and dark matter represent the coupling of the intensities of their respective gravitational fields, i.e., an interaction which has been instigated by $\gamma_{j k}^{i}$ and propagated by the curvature tensor in the deviation of geodesics. This shows that the interaction between ordinary and dark matter has been incorporated in the geometry of the spacetime due to curvature being an intrisic geometric property of space. Thus, the interaction of the gravitational field intensities is now being manifested in the Deviation Equation (16) and therefore in the tidal forces. This means that the geodesics we understand are deviated by the unified curvature including both dark and ordinary matter, instead of the geodesic motion in the ordinary spacetime as we have already noticed in rel. (11).

\subsection{Einstein Equations}

We shall now present the Riemann curvature tensor in its covariant form since it shall prove useful later on. By lowering the upper index in Equation $(12)^{6}$ we get:

$$
R_{a b c d}=R_{a b c d}^{(O)}+R_{a b c d}^{(D)}+\varrho_{a b c d}
$$

where,

$$
R^{(O)}{ }_{a b c d}=g^{(O)}{ }_{a e} R^{(O) e}{ }_{b c d}
$$

is ordinary matter's covariant Riemann tensor,

\footnotetext{
The lower-indices $[c d]$ denote an antisymmetrization (similarly, indices between parentheses shall denote symmetrization). One must be careful that for the unified space $(M, g)$ there is but one curvature tensor; the unified $R^{a}{ }_{b c d}$

For such operations we must always use the unified metric.
} 


$$
R^{(D)}{ }_{a b c d}=g^{(D)}{ }_{a e} R^{(D) e}{ }_{b c d}
$$

is dark matter's covariant curvature tensor and,

$$
\varrho_{a b c d}=g^{(D)}{ }_{a e} R^{(O) e}{ }_{b c d}+g^{(O)}{ }_{a e} R^{(D) e}{ }_{b c d}+g_{a e} r_{b c d}^{e}
$$

is the interaction part of the tensor.

By virtue of Equation (18), we can now find the Ricci curvature:

$$
R_{a b}=R_{a b}^{(O)}+R_{a b}^{(D)}+\xi_{a b}
$$

where,

$$
R_{a b}^{(O)}=g^{(O) c d} R^{(O)}{ }_{c a d b}
$$

is ordinary matter's Ricci curvature tensor,

$$
R_{a b}^{(D)}=g^{(D) c d} R_{c a d b}^{(D)}
$$

is dark matter's Ricci curvature tensor and,

$$
\xi_{a b}=R_{a b}-\left(R_{a b}^{(O)}+R_{a b}^{(D)}\right)
$$

is the interaction part of the Ricci tensor.

Using the form of the Ricci tensor given in Equation (22), we can find the Ricci scalar:

$$
R=R^{(O)}+R^{(D)}+\kappa
$$

where,

$$
R^{(O)}=g^{(O) a b} R_{a b}^{(O)}
$$

is ordinary matter's Ricci scalar,

$$
R^{(D)}=g^{(D) a b} R_{a b}^{(D)}
$$

is dark matter's Ricci scalar and,

$$
\kappa=R-\left(R^{(O)}+R^{(D)}\right)
$$

is the interaction part of the Ricci scalar.

The actions of the sectoral ordinary and dark matter gravitational fields and matter are, respectively:

$$
S_{O}=\int\left(\frac{1}{16 \pi} R^{(O)}+\mathcal{L}_{M_{O}}\right) \sqrt{-g^{(O)}} d^{4} x
$$

and

$$
S_{D}=\int\left(\frac{1}{16 \pi} R^{(D)}+\mathcal{L}_{M_{D}}\right) \sqrt{-g^{(D)}} d^{4} x
$$

where $\mathcal{L}_{M_{O}}$ and $\mathcal{L}_{M_{D}}$ describe the sectoral ordinary and dark matter fields, $g^{(O)}=\operatorname{det} g^{(O)}{ }_{i j}$ and $g^{(D)}=\operatorname{det} g^{(D)}{ }_{i j}, M_{O}$ and $M_{D}$ are the ordinary and dark matter masses, respectively ${ }^{7}$. It must be

7 We will refrain from using specific Lagrangians neither for the ordinary nor for the dark matter sector due to the existence of a plethora of potential Lagrangians for ordinary matter and a possible need for a complicated Lagrangian in order to effectively reproduce the dark sector phenomenology [38]. 
noted that with the addition of dark matter to our model, one must consider the action of the unified gravitational field and of the total matter ${ }^{8}$, which shall be

$$
S=\int\left(\frac{1}{16 \pi} R+\mathcal{L}_{M}\right) \sqrt{-g} d^{4} x
$$

where $\mathcal{L}_{\mathcal{M}}$ describes the unified matter fields, $g=\operatorname{det} g_{i j}$ and the total matter is $M=M_{O}+M_{D}$. By varying this action and by following the standard procedure of deriving the field equations we obtain the following unified Einstein equations:

$$
R_{a b}+\Lambda g_{a b}-\frac{1}{2} R g_{a b}=8 \pi T_{a b}
$$

where $\Lambda$ is the cosmological constant and $T_{a b}$ is the unified energy-momentum tensor. Using Equations (2), (22) and (26) in (33), we obtain the Einstein equation in the following modified form given below ${ }^{9}$ :

$$
\begin{aligned}
\left(R_{a b}^{(O)}+\Lambda g_{a b}^{(O)}-\frac{1}{2} R_{a b}^{(O)} g_{a b}^{(O)}\right) & \left(R_{a b}^{(D)}+\Lambda g_{a b}^{(D)}-\frac{1}{2} R^{(D)} g_{a b}^{(D)}\right) \\
& +\frac{1}{2}\left(2 \xi_{a b}-\kappa g_{a b}-R^{(O)} g_{a b}^{(D)}-R^{(D)} g_{a b}^{(O)}\right)=8 \pi G T_{a b}
\end{aligned}
$$

where,

$$
\begin{aligned}
& R_{a b}^{(O)}+\Lambda g^{(O)}{ }_{a b}-\frac{1}{2} R^{(O)} g_{a b}^{(O)}=8 \pi G T^{(O)}{ }_{a b} \\
& R_{a b}^{(D)}+\Lambda g^{(D)}{ }_{a b}-\frac{1}{2} R^{(D)} g^{(D)}{ }_{a b}=8 \pi G T^{(D)}{ }_{a b}
\end{aligned}
$$

and

$$
2 \xi_{a b}-\kappa g_{a b}-R^{(O)} g_{a b}^{(D)}-R^{(D)} g_{a b}^{(O)}=16 \pi G \tau_{a b}
$$

One can clearly observe that Equation (35) is the Einstein equation for ordinary matter, with $T^{(O)}{ }_{a b}$ being the energy-momentum tensor of ordinary matter. The corresponding relation for dark matter is also true as can be seen from Equation (36), where $T_{a b}^{(D)}$ plays the role of dark matter's energy-momentum tensor.

On the other hand, Equation (37) is a relation containing terms that hint towards gravitational interaction between ordinary and dark matter. The tensorial object $\tau_{a b}$ acts as the interaction part of the unified energy-momentum tensor since

$$
T_{a b}=T_{a b}^{(O)}+T_{a b}^{(D)}+\tau_{a b}
$$

As we shall see, the Einstein Equations (34) yield interesting results pertaining to the gravitational interaction of dark and ordinary matter. By taking the trace of Equation (34) we obtain the following result:

$$
\kappa=4 \Lambda-8 \pi T-R^{(O)}-R^{(D)}
$$

where $T$ is the trace of the unified energy-momentum tensor.

\subsection{Raychaudhuri Equation}

The Raychaudhuri equation which constitutes an extension of the geodesic deviation equation, plays a significant role in relativity theory and cosmology due to its connection with singularities [39-42].

8 One can clearly see that $S \neq S_{O}+S_{D}$.

9 We assume $c=1$. 
It governs the behavior and evolution of a family of test particles moving in world lines with given certain variables $(\theta, \sigma, \omega)$, where $\theta$ measures the rate of change of the cross-sectional area enclosing a family of geodesics ${ }^{10}$, the shear $\sigma$ measures anisotropy, and $\omega$ shows a rotation. The form of the equation depends on the geometry of the spacetime; if a geodesic motion of a family of test particles converges, the expansion $\theta$ is negative, and diverges when $\theta$ is positive.

We shall henceforth consider that both ordinary and dark matter can be considered as perfect fluids, each individually and together as a unified matter perfect fluid comprising the sum of ordinary and dark matter perfect fluids [43-45]. The stress-energy tensor for the perfect fluid distribution of the unified matter (ordinary and dark) with pressure $p$ and energy density $\rho$ falling under its own gravity with 4-velocity $u^{a}$ is then known to be:

$$
T_{a b}=(\rho+p) u_{a} u_{b}+p g_{a b}
$$

However, the unified fluid is composed of two-perfect fluids [46,47] the stress-energy tensor and shall therefore assume the following form $[48,49]$ :

$$
T_{a b}=\left(\rho^{(O)}+p^{(O)}\right) u_{a}^{(O)} u_{b}^{(O)}+p^{(O)} g_{a b}+\left(\rho^{(D)}+p^{(D)}\right) u_{a}^{(D)} u_{b}^{(D)}+p^{(D)} g_{a b}
$$

where $p^{(O)}, p^{(D)}$ is the pressure of the ordinary and dark matter fluids with energy density $\rho^{(O)}$ and $\rho^{(D)}$ and 4 -velocity $u_{a}^{(O)}$ and $u_{a}^{(D)}$, respectively.

Using our assumption (2) Equation (41) becomes:

$$
\begin{aligned}
T_{a b}=\left(\rho^{(O)}+p^{(O)}\right) u_{a}^{(O)} u_{b}^{(O)} & +p^{(O)} g^{(O)}{ }_{a b}+\left(\rho^{(D)}+p^{(D)}\right) u_{a}^{(D)} u_{b}^{(D)} \\
& +p^{(D)} g^{(D)}{ }_{a b}+p^{(O)} g^{(D)}{ }_{a b}+p^{(D)} g^{(O)}{ }_{a b}
\end{aligned}
$$

One can therefore see that for a perfect fluid and in accordance with Equation (38)

$$
\begin{array}{r}
T^{(O)}{ }_{a b}=\left(\rho^{(O)}+p^{(O)}\right) u_{a}^{(O)} u_{b}^{(O)}+p^{(O)} g^{(O)}{ }_{a b} \\
T_{a b}^{(D)}=\left(\rho^{(D)}+p^{(D)}\right) u_{a}^{(D)} u_{b}^{(D)}+p^{(D)} g^{(D)}{ }_{a b} \\
\tau_{a b}=p^{(O)} g^{(D)}{ }_{a b}+p^{(D)} g^{(O)}{ }_{a b}
\end{array}
$$

We further define the following four operators acting on a vector, e.g., $u^{a}$ (or similarly on a tensor) of $(M, g)$, as follows:

$$
u_{\mid k}^{a}=\frac{\partial u^{a}}{\partial x^{k}}+u^{b} \Gamma_{b k}^{(O) a}
$$

is the covariant derivative with respect to the ordinary matter subspace, in other words, the covariant derivative of a space comprised of purely ordinary matter ${ }^{11}$.

Similarly,

$$
u_{\| k}^{a}=\frac{\partial u^{a}}{\partial x^{k}}+u^{b} \Gamma_{b k}^{(D) a}
$$

is the covariant derivative w.r.t. the dark matter subspace ${ }^{12}$.

$$
u_{\backslash k}^{a}=\frac{\partial u^{a}}{\partial x^{k}}-u^{b} \gamma_{b k}^{a}
$$

\footnotetext{
10 The same apply to any curves in general.

11 This is true only if the vector (or tensor) acted upon belongs to the space with metric $g^{(O)}$.

12 This is true only if the vector (or tensor) acted upon belongs to the space with metric $g^{(D)}$.
} 
will be an operation that resembles a covariant derivative but uses the interaction symbols, $\gamma_{b c}^{a}{ }^{13}$. Finally, the unified space covariant derivative is given by ${ }^{14}$ :

$$
u_{; k}^{a}=\frac{\partial u^{a}}{\partial x^{k}}+u^{b} \Gamma_{b k}^{a}=u^{a}{ }_{\mid k}+u_{\| k}^{a}-u_{\backslash k}^{a}
$$

Using this covariant derivative in the rest system of a perfect matter fluid (40) the conservation of the energy-momentum tensor is considered

$$
T_{; b}^{a b}=0
$$

Assuming that the velocity $u$ corresponding to the total matter fluid (ordinary and dark) is given by $u=u^{(O)}+u^{(D)}$, the Raychaudhuri equations shall be [39]:

$$
\dot{\theta}=-2\left(\sigma^{2}-\omega^{2}\right)-\frac{1}{3} \theta^{2}-R_{a b} u^{a} u^{b}+\dot{u}^{a} ; a
$$

where $2 \sigma^{2}=\sigma_{a b} \sigma^{a b}, 2 \omega^{2}=\omega_{a b} \omega^{a b}$.

The vorticity tensor, $\omega_{a b}=u_{[a ; b]}-\dot{u}_{[a} u_{b]}$, is given by

$$
\omega_{a b}=\omega_{a b}^{(O)}+\omega_{a b}^{(D)}-w_{a b}
$$

where

$$
\begin{aligned}
\omega_{a b}^{(O)} & =u^{(O)}{ }_{[a \mid b]}-\dot{u}^{(O)}{ }_{[a} u_{b]}^{(O)}{ }_{b]} \\
\omega^{(D)}{ }_{a b} & =u_{[a|| b]}^{(D)}-\dot{u}^{(D)}{ }_{[a} u^{(D)}{ }_{b]}
\end{aligned}
$$

are the vorticity tensors of ordinary and dark matter respectively, and

$$
w_{a b}=u_{[a \backslash b]}-u_{[a|| b]}^{(O)}-u_{[a \mid b]}^{(D)}+\dot{u}_{[a}^{(O)} u_{b]}^{(D)}+\dot{u}_{[a}^{(D)} u_{b]}^{(O)}
$$

is the interaction part of the vorticity tensor.

The unified expansion scalar, $\theta=u^{k} ; k$ assumes the form

$$
\theta=\theta^{(O)}+\theta^{(D)}-\vartheta
$$

where

$$
\begin{gathered}
\theta^{(O)}=u_{\mid k}^{k} \\
\theta^{(D)}=u^{k}{ }_{|| k}
\end{gathered}
$$

are the expansion scalars of ordinary and dark matter and

$$
\vartheta=u_{\| k}^{(O) k}+u_{\mid k}^{(D) k}-u_{\backslash k}^{k}
$$

is the interaction part of the expansion scalar.

13 This operation does not constitute a covariant derivative as the symbols $\gamma_{b c}^{a}$ are not proper Christoffel symbols and there is no corresponding geometric space.

14 One must be careful that only $u^{a}{ }_{; k}$ is the covariant derivative of $u^{a} \in(M, g)$; all other operations defined before represent arbitrary operators in the framework of the unified space and can only be treated otherwise if we restrict our study in the corresponding subspaces. 
The projection tensor, $h_{a b}=g_{a b}-u_{a} u_{b}$, has the following form:

$$
h_{a b}=h_{a b}^{(O)}+h_{a b}^{(D)}-u_{(a}^{(O)} u_{b)}^{(D)}
$$

where

$$
\begin{gathered}
h_{a b}^{(O)}=g_{a b}^{(O)}-u_{a}^{(O)} u_{b}^{(O)}, \\
h_{a b}^{(D)}=g^{(D)}{ }_{a b}-u_{a}^{(D)} u_{b}^{(D)}
\end{gathered}
$$

are the ordinary and dark matter projection tensors.

The unified shear tensor, $\sigma_{a b}=u_{(a ; b)}-\frac{1}{3} \theta h_{a b}-\dot{u}_{(a} u_{b)}$, shall be given by

$$
\sigma_{a b}=\sigma_{a b}^{(O)}+\sigma^{(D)}{ }_{a b}-s_{a b}
$$

where

$$
\begin{gathered}
\sigma^{(O)}{ }_{a b}=u^{(O)}{ }_{(a \mid b)}-\frac{1}{3} \theta^{(O)} h^{(O)}{ }_{a b}-\dot{u}^{(O)}{ }_{(a} u^{(O)}{ }_{b)} \\
\sigma^{(D)}{ }_{a b}=u^{(D)}{ }_{(a|| b)}-\frac{1}{3} \theta^{(D)} h^{(D)}{ }_{a b}-\dot{u}^{(D)}{ }_{(a} u^{(D)}{ }_{b)}
\end{gathered}
$$

are the shear tensors of ordinary matter and dark matter fluid respectively, and

$$
\begin{aligned}
s_{a b} & =u_{(a \backslash b)}-u^{(O)}{ }_{(a|| b)}-u^{(D)}{ }_{(a \mid b)}+\dot{u}^{(O)}{ }_{(a} u^{(D)}{ }_{b)}+\dot{u}^{(D)}{ }_{(a} u^{(O)}{ }_{b)}+ \\
& +\frac{1}{3}\left(\theta^{(O)}\left(h^{(D)}{ }_{a b}-u^{(O)}{ }_{(a} u^{(D)}{ }_{b)}\right)+\theta^{(D)}\left(h_{a b}^{(O)}-u^{(O)}{ }_{(a} u^{(D)}{ }_{b)}\right)-\vartheta h_{a b}\right)
\end{aligned}
$$

is the interaction part of the shear tensor. Finally, using the Einstein Equation (34) and in conjunction with relations (43)-(45) we can find that, apart from the unified Raychaudhuri scalar, $R_{a b} u^{a} u^{b}=$ $4 \pi\left(\rho+3 p-\frac{\Lambda}{4 \pi}\right)$, which includes ordinary and dark matter as well as their interaction, the following are also true:

$$
R_{a b}^{(O)} u^{(O) a} u^{(O) b}=4 \pi\left(\rho^{(O)}+3 p^{(O)}-\frac{\Lambda}{4 \pi}\right)
$$

is the Raychaudhuri scalar of ordinary matter and,

$$
R_{a b}^{(D)} u^{(D) a} u^{(D) b}=4 \pi\left(\rho^{(D)}+3 p^{(D)}-\frac{\Lambda}{4 \pi}\right)
$$

is the dark matter Raychaudhuri scalar.

By vitrue of relations (51), (52), (56) and (63) the expansion $\theta$, the shear $\sigma$ and the vorticity $\omega$, which in our model include contributions from dark matter and its interaction with ordinary matter, extend the Raychaudhuri equation which now takes into account the existence of extra mass in the form of dark matter. Since the Raychaudhuri equation plays a dominant role in the evolution of the universe, Equation (51) gives rise to a potential need to differentiate the existing considerations of singularities.

\subsection{Conformal Dark FLRW-Metric Structure}

In this section we consider an application of our model in cosmology. In particular, we shall use a FLRW metric structure for the ordinary matter sector and we will assume a conformal relation for the unified spacetime containing both ordinary and dark matter. Using a conformal factor $F\left(R^{(O)}\right)$ for the unified metric, we shall derive modified Friedmann equations and the continuity equation for a model which is described by $F(R)$ gravity [50,51]. We have chosen such an assumption for the metric of the unified space because studies in the conformal structure in the fields of General Relativity and 
Cosmology have given rise to viable theories related to dark matter and dark energy (e.g., [50-56]) and this framework has been seen to play a significant role as the angles in the light-cone structure are preserved [52]. Indeed, we shall further choose a particular class of $F\left(R^{(O)}\right)$ [53] and we shall see that the metric of the dark matter sector is also conformal with the ordinary matter FLRW metric structure; namely we assume that

$$
F\left(R^{(O)}\right)=R^{(O)}+f\left(R^{(O)}\right)
$$

where $F\left(R^{(O)}\right)$ and $f\left(R^{(O)}\right)$ are functions of the Ricci scalar of the ordinary matter sector $R^{(O)}$. The function $F\left(R^{(O)}\right)$ is the conformal factor of the unified metric and $f\left(R^{(O)}\right)$ serves as the conformal factor of the dark metric. This is seen as a direct consequence of the assumption (2) as

$$
g_{a b}=F^{\prime}\left(R^{(O)}\right) g_{a b}^{(O)}=\left(1+f^{\prime}\left(R^{(O)}\right)\right) g_{a b}^{(O)}=g_{a b}^{(O)}+f^{\prime}\left(R^{(O)}\right) g_{a b}^{(O)}
$$

where $F^{\prime}\left(R^{(O)}\right)=\frac{d F\left(R^{(O)}\right)}{d R^{(O)}}$ and $f^{\prime}\left(R^{(O)}\right)=\frac{d f\left(R^{(O)}\right)}{d R^{(O)}}$.

Alternatively one could start from the assumption that the dark metric is conformal with the ordinary FLRW metric with conformal factor $f\left(R^{(O)}\right)$ [52]; namely

$$
g^{(D)}{ }_{a b}=f^{\prime}\left(R^{(O)}\right) g^{(O)}{ }_{a b}
$$

and using the assumption (2) we find that

$$
g_{a b}=g_{a b}^{(O)}+g^{(D)}{ }_{a b}=\left(1+f^{\prime}\left(R^{(O)}\right)\right) g_{a b}^{(O)}
$$

Therefore the unified metric is also conformal with the ordinary FLRW metric if we assume a conformal factor $F\left(R^{(O)}\right)$ of the form given in (69).

Using this previous assumption in conjunction with relations (7), (22), (26) and (38) we can calculate the Christoffel symbols, the Ricci curvature as well as the energy momentum tensor ${ }^{15}$. From the Einstein Equation (34) we then derive the following modified Friedmann equations

$$
\begin{aligned}
& \left(\frac{\dot{a}}{a}\right)^{2}=\frac{8 \pi G \rho}{3} F^{\prime}-\frac{\kappa}{a^{2}}+\frac{1}{6} \Phi_{1}(t) \\
& \frac{\ddot{a}}{\alpha}=-\frac{4 \pi G(\rho+3 p)}{3} F^{\prime}+\frac{1}{6} \Phi_{2}(t)
\end{aligned}
$$

where $a$ is the scale factor, $\kappa= \pm 1,0, \Phi_{1}(t)=\phi^{t} \phi_{t}+\phi_{\mid t}^{t}+\phi_{t \mid t}-\frac{\left(\phi_{t}\right)^{2}}{2}, \Phi_{2}(t)=\phi^{t} \phi_{t}+\phi_{\mid t}^{t}-2 \phi_{t \mid t}+\left(\phi_{t}\right)^{2}$, $\phi_{t}=\partial_{t}\left[\ln \left[F^{\prime}\right]\right], \rho=\rho^{(O)}+\rho^{(D)}, p=p^{(O)}+p^{(D)}$ and the operator " " has been defined in (46).

The idea of inflation can be incorporated into the model of Friedmann equations by taking into account the Planck mass $m_{P l}=(8 \pi G)^{-1 / 2}[57,58]$. We can then write

$$
\begin{aligned}
& H^{2}=\frac{\rho}{3 m_{P l}} F^{\prime}-\frac{\kappa}{a^{2}}+\frac{1}{6} \Phi_{1}(t) \\
& \frac{\ddot{a}}{a}=-\frac{(\rho+3 p)}{6 m_{P l}} F^{\prime}+\frac{1}{6} \Phi_{2}(t)
\end{aligned}
$$

where $H=\dot{a} / a$ is the Hubble parameter. By virtue of the above relations we can see that the additional terms $\Phi_{i}(t), i=1,2$ constitute an extension of the Friedmann equations and may contribute to a differentiation to the accelerated expansion of the universe which is incorporated in the observations. In a spacetime where dark matter is absent, therefore $f^{\prime}=0$ or equivalently $F^{\prime}=1$, it can be easily

15 The velocities are also assumed to follow a conformal relation [54]. 
verified that $\Phi_{i}(t)$ also vanish and thus, we recover the usual form of the Friedmann equations for a FLRW ordinary matter space.

Taking into account that the energy momentum tensor of the ordinary sector is conserved, $T_{0 \mid \mu}^{(O) \mu}=0$, we can see that the energy momentum tensor of the unified two component cosmological fluid is also conserved and using the fact that $T_{0 ; \mu}^{\mu}=0$ we derive the following continuity equation

$$
\dot{\rho}+3(1+w) \rho H=0
$$

where we assume that the total pressure $p=w \rho$. This result is also compatible with the general conservation relation (50).

\section{Gravity on the Sasaki Tangent Bundle}

For the study of the tangent bundle we use a metric, $g$, on a Riemannian manifold $(M, g)$, along with its Levi-Civita connection, to construct a new natural Riemannian metric on the tangent bundle $T M$, called the Sasaki metric. The geometry of tangent bundles in general and particularly the Sasaki metric find a lot of applications in physics, especially in the study of gravity. This consideration extends the limits of conventional general relativity for a modified gravity approach, and provides the gravitational field with extra degrees of freedom. In particular, a Sasaki extension of spacetime constitutes the minimum metric generalization of spacetime in the framework of a tangent bundle of a four-dimensional spacetime.

\subsection{Deviation of Geodesics of a Sasaki Spacetime}

In this section, we shall derive the deviation of geodesics of a Sasaki spacetime with metric [31]

$$
d \sigma^{2}=g_{\alpha \beta}(x) d x^{\alpha} d x^{\beta}+g_{\alpha \beta}(x) D y^{\alpha} D y^{\beta}
$$

where,

$$
D y^{\alpha}=d y^{\alpha}+N_{\beta}^{\alpha}(x, y) d x^{\beta}
$$

$N^{\mu}{ }_{\alpha}(x, y)$ represents the "pre-Finsler non-linear connection", $g_{\alpha \beta}(x)$ is the Riemann metric tensor of the n-dimensional differentiable manifold (here $\mathrm{n}=4$ ) and both the horizontal and vertical part of the total metric (78) of the 2 -dimensional tangent bundle. $\alpha, \beta \in\{1,2, \ldots, n=4\}$.

Furthermore, for the purpose of our study, the non-linear connection is assumed to be

$$
N_{\alpha}^{\mu}=\Gamma_{\alpha \kappa}^{\mu} y^{\kappa}
$$

The metric (78) may, then be rewritten using the fundamental covariant metric tensor, $G_{i j}$ as

$$
d \sigma^{2}=G_{i j} d x^{i} d x^{j}
$$

where $i, j \in\{1,2, \ldots, 2 n=8\}$ and

$$
\begin{gathered}
G_{\alpha \beta}=g_{\alpha \beta}+g_{\mu \nu} \Gamma_{\rho \alpha}^{\mu} \Gamma^{\nu}{ }_{\kappa \beta} y^{\rho} y^{\kappa} \\
G_{\alpha(n+\beta)}=\Gamma_{\mu \alpha \beta} y^{\mu}
\end{gathered}
$$

and

$$
G_{(n+\alpha)(n+\beta)}=g_{\alpha \beta}
$$

where $\Gamma_{\mu \alpha \beta}$ and $\Gamma_{\rho \alpha}^{\mu}$ are Christoffel's symbols of the first and second kind of $M^{n}$ respectively. 
If $\bar{\Gamma}_{i j k}$ and $\bar{\Gamma}_{j k}^{i}$ are Christoffel's symbols of first and second kind, respectively, that correspond to the Sasaki tangent bundle $T\left(M^{n}\right)$ as calculated using the fundamental metric, then the geodesic equation is known to be

$$
\frac{d^{2} x^{i}}{d \sigma^{2}}+\bar{\Gamma}^{i}{ }_{j k} \frac{d x^{j}}{d \sigma} \frac{d x^{k}}{d \sigma}=0
$$

While it is usually more useful to express this equation in terms of quantities of $M^{n}$, for the present we shall content ourselves with using relation (85) instead, in order to avoid perplexing our equations.

If $x_{s}^{i}(t)$ is a two-parameter geodesic family with $t$ being the affine parameter and $s$ the selector parameter then we shall denote the tangent vectors as $V^{i}=\partial_{t} x^{i}, W^{i}=\partial_{s} x^{i}$ so that $\partial_{s} V^{i}=\partial_{t} W^{i}$. Moreover, let $X^{i}\left(x^{k}\right)$ be a vector field defined over a region of the subspace of $T\left(M^{n}\right)$ defined by the net of $x_{s}^{i}(t)$. Then we define the $\delta$-derivatives as:

$$
\frac{\delta X^{i}}{\delta t}=X_{; h}^{i} V^{h}=\left(\frac{\partial X^{i}}{\partial x^{h}}+\bar{\Gamma}_{h k}^{i} X^{k}\right) V^{h}
$$

and

$$
\frac{\delta X^{i}}{\delta s}=X_{; h}^{i} W^{h}=\left(\frac{\partial X^{i}}{\partial x^{h}}+\bar{\Gamma}_{h k}^{i} X^{k}\right) W^{h}
$$

Using the previous result in conjunction with the fact that the Christoffel's symbols are symmetric w.r.t. their suffixes, we get that

$$
\frac{\delta V^{i}}{\delta s}=\frac{\partial^{2} x^{i}}{\partial t \partial s}+\bar{\Gamma}_{h k}^{i} V^{k} W^{h}=\frac{\delta W^{i}}{\delta t}
$$

Following the standard procedure of deriving the deviation equations [59], given the previous relations, we find that

$$
\frac{\delta^{2} W^{i}}{\delta t^{2}}=\frac{\delta^{2} V^{i}}{\delta s \delta t}=\frac{\delta^{2} V^{i}}{\delta t \delta s}-K^{i}{ }_{h h k} V^{j} V^{h} W^{k}
$$

Finally, using Equation (85) we find the following geodesic deviation equation

$$
\frac{\delta^{2} W^{i}}{\delta t^{2}}=-K_{j h k}^{i} V^{j} V^{h} W^{k}
$$

where $K^{i}{ }_{j h k}$ is the curvature tensor of the tangent bundle. An abstract form of the curvature tensor is given by $[60,61]$. All the components of the curvature tensor $K_{j h k}^{i}$ are given explicitly in Appendix A.

\subsection{Dark Gravity on the Tangent Bundle}

Experimental research [32-36] suggests that a theory of dark gravity with extra dimensions may be necessary in order to effectively describe the total mass distribution in the universe. A first step towards such a geometric gravitational theory could be obtained by retaining our assumption (2) and expanding our Riemannian framework from Section 2 on the tangent bundle using an underlying Sasaki structure (78) for the total space of ordinary and dark matter.

First, taking into account relation (80) and using Equation (7) we find that

$$
N_{\alpha}^{\mu}=N_{\alpha}^{(O) \mu}+N_{\alpha}^{(D) \mu}+v^{\mu}{ }_{\alpha}
$$

where,

$$
N_{\alpha}^{(O) \mu}=\Gamma_{\alpha \kappa}^{(O) \mu} y^{\kappa}
$$

is the ordinary matter non-linear connection,

$$
N_{\alpha}^{(D) \mu}=\Gamma_{\alpha \kappa}^{(D) \mu} y^{\kappa}
$$


is dark matter's non-linear connection, and

$$
v_{\alpha}^{\mu}=\gamma_{\alpha \kappa}^{\mu} y^{\kappa}
$$

is the interaction part of the non-linear connection. $v^{\mu}{ }_{\alpha}$ acts as a correlation between the non-linear connections of ordinary and dark matter. Thus, in the framework of a Sasaki spacetime, $v^{\mu}{ }_{\alpha}$ plays the fundamental role of interconnecting the ordinary and dark matter sectors within the line-element. Such a connection between ordinary and dark matter is absent from the corresponding line-element of the Riemannian spacetime. It can therefore be concluded that a Sasaki spacetime involves a stronger interaction between the two component matter sectors which influences even the notion of arc-length as we shall also see below.

In virtue of relations (79) and (91), we obtain

$$
D y^{\mu}=D^{(O)} y^{\mu}+D^{(D)} y^{\mu}-\delta y^{\mu}
$$

where,

$$
\begin{aligned}
& D^{(O)} y^{\mu}=d y^{\mu}+N_{\alpha}^{(O) \mu}{ }_{\alpha}(x, y) d x^{\alpha} \\
& D^{(D)} y^{\mu}=d y^{\mu}+N_{\alpha}^{(D) \mu}(x, y) d x^{\alpha}
\end{aligned}
$$

and

$$
\delta y^{\mu}=d y^{\mu}-v^{\mu}{ }_{\alpha}(x, y) d x^{\alpha}
$$

In a Sasaki spacetime, the non-linear connection influences the gravitational potential giving rise to extra degrees of freedom in the internal structure of the spacetime in the form of $y$-dependence. As evidenced by relations (2), (78), (79) and (95), with the addition of dark matter on the tangent bundle of the spacetime, three types of line-elements can be presented:

$$
d \sigma^{2}=d \sigma_{O}^{2}+d \sigma_{D}^{2}+d \sigma_{I}^{2}
$$

where

$$
d \sigma_{O}^{2}=g^{(O)}{ }_{\alpha \beta} d x^{\alpha} d x^{\beta}+g^{(O)}{ }_{\alpha \beta} D^{(O)} y^{\alpha} D^{(O)} y^{\beta}
$$

is the line-element of the Sasaki tangent bundle of ordinary matter,

$$
d \sigma_{D}^{2}=g^{(D)}{ }_{\alpha \beta} d x^{\alpha} d x^{\beta}+g^{(D)}{ }_{\alpha \beta} D^{(D)} y^{\alpha} D^{(D)} y^{\beta}
$$

is the line-element of the Sasaki tangent bundle of dark matter, and

$$
d \sigma_{I}^{2}=g_{\alpha \beta} D y^{\alpha} D y^{\beta}-g^{(O)}{ }_{\alpha \beta} D^{(O)} y^{\alpha} D^{(O)} y^{\beta}-g^{(D)}{ }_{\alpha \beta} D^{(D)} y^{\alpha} D^{(D)} y^{\beta}
$$

is the interaction term of the line-element.

It can be seen that the non-linear connection, $N(x, y)$, plays a fundamental role in this approach, since it facilitates the introduction of three differentials (95) that produce extra interaction terms between ordinary and dark matter. In the base manifold $(M, g)$ of Section 2 , the introduction of dark matter extends the arc-length of the spacetime of ordinary matter in an additive way since one could write relation (1) as

$$
d s^{2}=d s_{O}^{2}+d s_{D}^{2}
$$

where

$$
d s_{O}^{2}=g^{(O)}{ }_{\alpha \beta} d x^{\alpha} d x^{\beta}
$$

is the line-element of an ordinary matter spacetime, and

$$
d s_{D}^{2}=g^{(D)}{ }_{\alpha \beta} d x^{\alpha} d x^{\beta}
$$


is the line-element of a dark matter spacetime. Such "trivial" extension is not possible in the Sasaki tangent bundle because of the non-linear connection introducing extra interaction that influences even the arc-length itself.

We have seen that the geodesics are provided by the condensed relation (85) which is known to be expanded in the following two equations [31]:

$$
\begin{aligned}
\frac{d^{2} x^{\mu}}{d \sigma^{2}}+\Gamma^{\mu}{ }_{\nu \rho} \frac{d x^{\nu}}{d \sigma} \frac{d x^{\rho}}{d \sigma} & =R^{\mu}{ }_{\nu \alpha \beta} \frac{d x^{\nu}}{d \sigma} y^{\alpha} \frac{D y^{\beta}}{d \sigma} \\
\frac{D^{2} y^{\mu}}{d \sigma^{2}} & =0
\end{aligned}
$$

where $\Gamma^{\mu}{ }_{v \rho}$ and $R^{\mu}{ }_{v \alpha \beta}$ are the Christoffel symbols and Riemann curvature tensor, respectively, corresponding to the base manifold $(M, g)$ as discussed in Section 2, and

$$
\begin{gathered}
\frac{D y^{\mu}}{d \sigma}=\frac{d y^{\mu}}{d \sigma}+\Gamma^{\mu}{ }_{\nu \rho} y^{\nu} \frac{d x^{\rho}}{d \sigma} \\
\frac{D^{2} y^{\mu}}{d \sigma^{2}}=\frac{d^{2} y^{\mu}}{d \sigma^{2}}+\frac{d}{d \sigma}\left\{\Gamma^{\mu}{ }_{\nu \rho} y^{\nu} \frac{d x^{\rho}}{d \sigma}\right\}+\Gamma^{\mu}{ }_{\alpha \beta} \frac{D y^{\alpha}}{d \sigma} \frac{d x^{\beta}}{d \sigma}
\end{gathered}
$$

The expansion of the condensed geodesic Equation (85) gave rise to two geodesic equations. The horizontal geodesic (106) can be physically interpreted as the generalization of the corresponding Riemannian geodesic curves on the tangent bundle. The expanded family of geodesic curves described by (106) shall be further examined below. On the other hand, the physical interpretation of the vertical Equation (107) remains an open question and although we shall continue to provide the vertical equations, we shall limit our present work to the physical study of the horizontal geodesics.

If we compare the Riemannian setting with the Sasaki tangent bundle, we can see from relation (106) that the class of curves that have the geodesic property has now been expanded. On $(T M, G)$ we can distinguish two geodesic families the first of which is obtained by lifting a geodesic of $(M, g)$ on $T M$. In that case the expanded form of the geodesic equations reduces as [31]

$$
\begin{gathered}
\frac{d^{2} x^{\mu}}{d \sigma^{2}}+\Gamma_{\nu \rho}^{\mu} \frac{d x^{v}}{d \sigma} \frac{d x^{\rho}}{d \sigma}=0 \\
\frac{D y^{\mu}}{d \sigma}=0
\end{gathered}
$$

In this case, with reference to the base manifold $M$ of $T M$, an observer shall continue to perceive a state of rest since Equation (110) coincides with their notion of a Riemannian geodesic. We will henceforth call an observer limited to perceiving only a Riemannian manifold occupied by ordinary matter i.e., neglecting dark matter and ignoring the existence of any higher-dimensional structure, a constrained observer. In essence, "constrained" observers think that they are experiencing the submanifold $\left(M^{(O)}, g^{(O)}\right)$ of the base manifold, thereby perceiving anything deviating from their notion of a Riemannian structure as due to external forces or effects. Such interpretation is compatible with the idea of an apparent metric as proposed by [52].

The second family of geodesics on the Sasaki tangent bundle is comprised by curves that are not obtained by lifting geodesics of $M$. In that case Equation (106) cannot be further reduced. This causes a constrained observer to perceive apparent pseudo-coupling forces of the gravitational field with the velocity field of $T M$, preventing them from understanding that curve as a geodesic. The r.h.s. part of Equation (106) seemingly disagrees with the notion of a Riemannian geodesic. However, this effect is only due to the observer's inability to perceive TM. 
By virtue of relations (95) and (108) we have that:

$$
\frac{D y^{\mu}}{d \sigma}=\frac{D^{(O)} y^{\mu}}{d \sigma}+\frac{D^{(D)} y^{\mu}}{d \sigma}-\frac{\delta y^{\mu}}{d \sigma}
$$

where

$$
\begin{aligned}
\frac{D^{(O)} y^{\mu}}{d \sigma} & =\frac{d y^{\mu}}{d \sigma}+\Gamma^{(O) \mu}{ }_{\nu \rho} y^{v} \frac{d x^{\rho}}{d \sigma} \\
\frac{D^{(D)} y^{\mu}}{d \sigma} & =\frac{d y^{\mu}}{d \sigma}+\Gamma^{(D) \mu}{ }_{v \rho} y^{\nu} \frac{d x^{\rho}}{d \sigma} \\
\frac{\delta y^{\mu}}{d \sigma} & =\frac{d y^{\mu}}{d \sigma}-\gamma^{\mu}{ }_{\nu \rho} y^{\nu} \frac{d x^{\rho}}{d \sigma}
\end{aligned}
$$

Using Equations (109) and (111) we get:

$$
\frac{D^{2} y^{\mu}}{d \sigma^{2}}=\frac{D^{(O) 2} y^{\mu}}{d \sigma^{2}}+\frac{D^{(D) 2} y^{\mu}}{d \sigma^{2}}+\frac{\delta^{2} y^{\mu}}{d \sigma^{2}}+\frac{D^{(O)}}{d \sigma}\left\{\frac{D^{(D)} y^{\mu}}{d \sigma}-\frac{\delta y^{\mu}}{d \sigma}\right\}+\frac{D^{(D)}}{d \sigma}\left\{\frac{D^{(O)} y^{\mu}}{d \sigma}-\frac{\delta y^{\mu}}{d \sigma}\right\}-\frac{\delta}{d \sigma}\left\{\frac{D^{(O)} y^{\mu}}{d \sigma}+\frac{D^{(D)} y^{\mu}}{d \sigma}\right\}
$$

where

$$
\begin{gathered}
\frac{D^{(O) 2} y^{\mu}}{d \sigma^{2}}=\frac{d^{2} y^{\mu}}{d \sigma^{2}}+\frac{d}{d \sigma}\left\{\Gamma_{{ }_{\nu}}^{(O) y^{\nu}} \frac{d x^{\rho}}{d \sigma}\right\}+\Gamma_{\alpha \beta}^{(O) \mu} \frac{D^{(O)} y^{\alpha}}{d \sigma} \frac{d x^{\beta}}{d \sigma} \\
\frac{D^{(D) 2} y^{\mu}}{d \sigma^{2}}=\frac{d^{2} y^{\mu}}{d \sigma^{2}}+\frac{d}{d \sigma}\left\{\Gamma_{{ }_{\nu \rho}}^{(D) \mu} \frac{d x^{\rho}}{d \sigma}\right\}+\Gamma_{\alpha \beta}^{(D) \mu} \frac{D^{(D)} y^{\alpha}}{d \sigma} \frac{d x^{\beta}}{d \sigma} \\
\frac{\delta^{2} y^{\mu}}{d \sigma^{2}}=\frac{d^{2} y^{\mu}}{d \sigma^{2}}+\frac{d}{d \sigma}\left\{\gamma^{\mu}{ }_{\nu \rho} y^{\nu} \frac{d x^{\rho}}{d \sigma}\right\}+\gamma_{\alpha \beta}^{\mu} \frac{\delta y^{\alpha}}{d \sigma} \frac{d x^{\beta}}{d \sigma}
\end{gathered}
$$

From the point of view of an observer limited to the ordinary matter subspace relations (6) and (11) show that the geodesic equations in the Riemannian setting are perturbed when dark matter is taken into account. Let us now assume a test particle moving along geodesics on the tangent bundle in a Sasaki spacetime. In analogy to the Riemannian case the geodesics in higher dimensions will deviate from the previously thought geodesic motion due to the presence of dark matter and its interaction with ordinary matter. In this case, using our previous results in conjunction with relations (7), (12) and (111) in Equations (106) and (107), the unified form of the corresponding geodesic equation will be given by the following:

$$
\frac{d^{2} x^{\mu}}{d \sigma^{2}}+\left(\Gamma_{\nu \rho}^{(O) \mu}+\Gamma_{\nu \rho}^{(D) \mu}+\gamma^{\mu}{ }_{\nu \rho}\right) \frac{d x^{v}}{d \sigma} \frac{d x^{\rho}}{d \sigma}=\left(R_{\nu \alpha \beta}^{(O) \mu}+R_{\nu \alpha \beta}^{(D) \mu}+r^{\mu}{ }_{\nu \alpha \beta}\right) \frac{d x^{v}}{d \sigma} y^{a}\left(\frac{D^{(O)} y^{b}}{d \sigma}+\frac{D^{(D)} y^{b}}{d \sigma}-\frac{\delta y^{b}}{d \sigma}\right)
$$

and

$$
\frac{D^{(O) 2} y^{\mu}}{d \sigma^{2}}+\frac{D^{(D) 2} y^{\mu}}{d \sigma^{2}}+\frac{\delta^{2} y^{\mu}}{d \sigma^{2}}=-\frac{D^{(O)}}{d \sigma}\left\{\frac{D^{(D)} y^{\mu}}{d \sigma}-\frac{\delta y^{\mu}}{d \sigma}\right\}-\frac{D^{(D)}}{d \sigma}\left\{\frac{D^{(O)} y^{\mu}}{d \sigma}-\frac{\delta y^{\mu}}{d \sigma}\right\}+\frac{\delta}{d \sigma}\left\{\frac{D^{(O)} y^{\mu}}{d \sigma}+\frac{D^{(D)} y^{\mu}}{d \sigma}\right\}
$$

As we have discussed, the expanded family of geodesics of a Sasaki spacetime includes curves that can be obtained by lifting geodesics of the base manifold. In this case, from the perspective of a constrained observer who takes into account only the ordinary matter sector of the Riemannian space $(M, g)$, the test particle moves along a curve that deviates from their expected Riemannian geodesics due to the presence of dark pseudo-forces ${ }^{16}$. If however, the test particle at rest happens to be moving along a Sasaki geodesic that cannot be obtained by such a lift, then the constrained observer will detect, in addition to the dark pseudo-forces, the pseudo-coupling forces given by the r.h.s. part of

16 These are the same $F_{(D)}^{i}$ as in Equation (11). 
Equation (119). Therefore, in a Sasaki spacetime a constrained observer shall always observe deviated curves as is the case with a Riemannian spacetime. The difference between a Riemannian and a Sasaki setting lies in the observation of the pseudo-coupling forces which may or may not be detected depending on the geodesic.

By virtue of relation (A1) it can be shown that

$$
K_{j h k}^{i}=K_{j h k}^{(O) i}+K_{j h k}^{(D) i}+\kappa_{j h k}^{i}
$$

where $K^{(O) i}{ }_{j h k^{\prime}} K^{(D) i}{ }_{j h k^{\prime}}$ are the Sasaki curvature tensors of ordinary and dark matter, respectively, and $\kappa^{i}{ }_{j h k}=K_{j h k}^{i}-K_{j h k}^{(O) i}-K^{(D) i}{ }_{j h k}$.

Using relation (90) with the above result we get

$$
\frac{\delta^{2} W^{i}}{\delta t^{2}}=-\left(K_{j h k}^{(O) i}+K^{(D) i}{ }_{j h k}+\kappa_{j h k}^{i}\right) V^{j} V^{h} W^{k}
$$

As we have seen from relation (A1), the curvature tensor, $K$, has been modified in higher dimensions due to the geometry of the Sasaki tangent bundle. Consequently, we can conclude that the gravitational field has also been influenced by the higher-dimensional metric structure of the spacetime, i.e., the Sasaki tangent bundle, since gravity is intertwined with the notion of curvature. The gravitational field has been endowed with extra degrees of freedom and this has been incorporated in the tidal forces (122) by means of the generalized curvature tensor. As a result, the deviation of the 4-dimensional free motion of nearby particles (or clusters thereof, e.g., galaxies) (16) can be differentiated from its higher-dimensional counterpart because of the form of the curvature tensor which is an intrinsic geometric property of the spacetime and therefore independent of the relative observer. Therefore, the resulting dark tidal forces manifest both the addition of extra matter and interaction in the form of dark matter as well as the higher-dimensional geometric structure of the spacetime and the subsequent extra degrees of freedom of the gravitational field.

The previous result is of great importance for cosmology. For instance, a positive curvature of a Riemannian spacetime which is connected with converging neighbouring geodesics (Jacobi field) may correspond to a negative curvature on the Sasaki tangent bundle. Hence, taking into account the higher-dimensional structure could potentially reveal that what we initially thought of as convergent is in reality divergent, and vice-versa, due to extra forces stemming from the geometry of the spacetime.

\section{Concluding Remarks}

Motivated by observational results, we laid the foundations for a geometric theory of dark gravity in relation to the ordinary gravitational field in the framework of a "unified" space. By extending the already existing notions of geometry that apply for ordinary matter, we used them in the case of dark gravity. In particular, in this work we examined the unified gravitational field of ordinary and dark matter in a Riemannian metric framework deriving the geodesic equations and their deviation, as well as the Einstein and Raychaudhuri equations. Furthermore, by extending our geometric methods on the tangent bundle we presented a unified dark and ordinary gravity on a Sasaki spacetime.

The geometric methods used throughout this paper gave rise to the concept of dark forces which could play a role in offsetting the centrifugal orbital tendency of the galaxies. In particular, the gravitational influence of dark matter generated modified geodesic curves that seemingly deviate from the Riemannian geodesic notion due to the presence of dark pseudo-forces (11) and (119) which appear to dominate the deviated geodesic motion. The extension of the spacetime on the tangent bundle also caused the potential emergence of the pseudo-coupling forces (119) due to the inability of the observer to perceive the higher dimensional structure. Both of these cases are deemed as pseudo-forces because they are the product of a constrained observer. The third kind of dark forces however, the dark tidal forces (16) and (122), result from an intrinsic geometric property of the 
spacetime; the dark curvature. This shows that the gravitational interaction between ordinary and dark matter has been incorporated in the geometry of the spacetime and therefore, dark tidal forces exist independent of the observer.

With the introduction of dark matter to the Riemannian spacetime setting, the Einstein and Raychaudhuri equations were modified to account for the existence of extra matter and thus, for extra gravitational interaction. These equations could provide invaluable information concerning the interaction of dark and ordinary matter and hence provide details about the internal structure of the unified space. The modification of these equations due to dark matter resulted in modified Friedmann Equations (73)-(76) as examined using a conformal metric structure for the dark matter sector. The study of inflation and the cosmological implications of these relations in the evolution of the universe as well as the application of other models of dark metric structure could constitute the subject of further research.

Overall, the existence of dark matter calls into question the concept of geodesic motion. Indeed the appearance of pseudo-force fields dominating the motion of test particles causes a constrained observer to conclude that the motion is not geodesic and that the ordinary matter spacetime has been externally perturbed by dark matter. Nonetheless, a closer look at the overall structure of the spacetime reveals that those apparent forces exist only relative to the point of view of a particular observer and reaffirms the geodesic property of the curves.

Ultimately, we consider that the use of geometric methods in the formulation of a gravitational theory that includes dark matter could form a theoretical basis upon which observational results could be interpreted. The contribution of dark energy in the framework of this theoretical effort remains an even greater question for future research.

Author Contributions: Both authors contributed equally. All authors have read and agreed to the published version of the manuscript.

Funding: We have received no funding for this project.

Conflicts of Interest: The authors declare no conflict of interest.

\section{Appendix A}

The Curvature Tensor of a Sasaki Tangent Bundle

Using the Christoffel symbols $\bar{\Gamma}^{i}{ }_{j k}$ as given by S. Sasaki in relation (7.4) in [31] and after long calculations, it can be seen that the curvature tensor of a $2 n$-dimensional Sasaki tangent bundle $(T M, G)$ is

$$
\begin{aligned}
& K_{(n+\alpha)(n+\beta)(n+\gamma)}^{i}=0 \\
& K_{(n+\alpha)(n+\beta) \gamma}^{\delta}=\frac{1}{2} \frac{\partial}{\partial x^{(n+\beta)}}\left[R_{\gamma \alpha \lambda}^{\delta} y^{\lambda}\right]+\frac{1}{4} R_{\epsilon \beta \mu}^{\delta} R_{\gamma \alpha \nu}^{\epsilon} y^{\mu} y^{\nu} \\
& K_{(n+\alpha)(n+\beta) \gamma}^{(n+\delta)}=\frac{\partial}{\partial x^{(n+\beta)}}\left[\Gamma_{\alpha \gamma}^{\delta}-\frac{1}{2} \Gamma_{\mu \lambda}^{\delta} R_{\gamma \alpha \nu}^{\lambda} y^{\mu} y^{\nu}\right]+\frac{1}{2}\left[\Gamma_{\beta \epsilon}^{\delta}-\frac{1}{2} \Gamma_{\mu \lambda}^{\delta} R_{\epsilon \beta \nu}^{\lambda} y^{\mu} y^{\nu}\right] R_{\gamma \alpha \kappa}^{\epsilon} y^{\kappa} \\
& K_{(n+\alpha) \beta \gamma}^{\delta}=\frac{1}{2} \frac{\partial}{\partial x^{\beta}}\left[R_{\gamma \alpha \lambda}^{\delta} y^{\lambda}\right]-\frac{1}{2} \frac{\partial}{\partial x^{\gamma}}\left[R_{\beta \alpha \lambda}^{\delta} y^{\lambda}\right]+\frac{1}{2}\left[\Gamma_{\beta \epsilon}^{\delta}+\frac{1}{2}\left(R^{\delta}{ }_{\epsilon \kappa \mu} \Gamma^{\kappa}{ }_{\nu \beta}+R_{\beta \kappa \mu}^{\delta} \Gamma^{\kappa}{ }_{v \epsilon}\right) y^{\mu} y^{\nu}\right] R_{\gamma \alpha \lambda}^{\epsilon} y^{\lambda} \\
& -\frac{1}{2}\left[\Gamma^{\delta}{ }_{\gamma \epsilon}+\frac{1}{2}\left(R^{\delta}{ }_{\epsilon \kappa \mu} \Gamma^{\kappa}{ }_{\nu \gamma}+R^{\delta}{ }_{\gamma \kappa \mu} \Gamma^{\kappa}{ }_{\nu \epsilon}\right) y^{\mu} y^{v}\right] R_{\beta \alpha \lambda}^{\epsilon} y^{\lambda}+\frac{1}{2}\left[\Gamma_{\alpha \gamma}^{\epsilon}-\frac{1}{2} \Gamma_{\mu \kappa}^{\epsilon} R_{\gamma \alpha \nu}^{\kappa} y^{\mu} y^{v}\right] R^{\delta}{ }_{\beta \epsilon \lambda} y^{\lambda} \\
& -\frac{1}{2}\left[\Gamma_{\alpha \beta}^{\epsilon}-\frac{1}{2} \Gamma_{\mu \kappa}^{\epsilon} R_{\beta \alpha \nu}^{\kappa} y^{\mu} y^{v}\right] R_{\gamma \in \lambda}^{\delta} y^{\lambda}
\end{aligned}
$$




$$
\begin{aligned}
& K_{(n+\alpha) \beta \gamma}^{(n+\delta)}=\frac{\partial}{\partial x^{\beta}}\left[\Gamma_{\alpha \gamma}^{\delta}-\frac{1}{2} \Gamma_{\mu \lambda}^{\delta} R_{\gamma \alpha \nu}^{\lambda} y^{\mu} y^{\nu}\right]-\frac{\partial}{\partial x^{\gamma}}\left[\Gamma_{\alpha \beta}^{\delta}-\frac{1}{2} \Gamma_{\mu \lambda}^{\delta} R_{\beta \alpha \nu}^{\lambda} y^{\mu} y^{\nu}\right] \\
& +\frac{1}{4} R_{\gamma \alpha \xi}^{\epsilon} y^{\xi}\left[\left(R_{\beta \lambda \epsilon}^{\delta}+R_{\epsilon \lambda \beta}^{\delta}+2 \frac{\partial \Gamma_{\beta \epsilon}^{\delta}}{\partial x^{\lambda}}\right) y^{\lambda}+\Gamma_{\nu \kappa}^{\delta}\left(R_{\epsilon \mu \eta}^{\kappa} \Gamma_{\lambda \beta}^{\eta}+R_{\beta \mu \eta}^{\kappa} \Gamma_{\lambda \epsilon}^{\eta}\right) y^{\lambda} y^{\mu} y^{v}\right] \\
& +\left[\Gamma_{\epsilon \beta}^{\delta}-\frac{1}{2} \Gamma_{\mu \kappa}^{\delta} R_{\beta \epsilon \nu}^{\kappa} y^{\mu} y^{\nu}\right]\left[\Gamma_{\alpha \gamma}^{\epsilon}-\frac{1}{2} \Gamma_{\lambda \eta}^{\epsilon} R_{\gamma \alpha \xi}^{\eta} y^{\lambda} y^{\xi}\right] \\
& -\frac{1}{4} R_{\beta \alpha \xi}^{\epsilon} y^{\xi}\left[\left(R_{\gamma \lambda \epsilon}^{\delta}+R_{\epsilon \lambda \gamma}^{\delta}+2 \frac{\partial \Gamma_{\gamma \epsilon}^{\delta}}{\partial x^{\lambda}}\right) y^{\lambda}+\Gamma_{\nu \mathcal{K}}^{\delta}\left(R_{\epsilon \mu \eta}^{\kappa} \Gamma_{\lambda \gamma}^{\eta}+R_{\gamma \mu \eta}^{\kappa} \Gamma_{\lambda \epsilon}^{\eta}\right) y^{\lambda} y^{\mu} y^{\nu}\right] \\
& -\left[\Gamma_{\epsilon \gamma}^{\delta}-\frac{1}{2} \Gamma_{\mu \kappa}^{\delta} R_{\gamma \epsilon \nu}^{\kappa} y^{\mu} y^{\nu}\right]\left[\Gamma_{\alpha \beta}^{\epsilon}-\frac{1}{2} \Gamma_{\lambda \eta}^{\epsilon} R_{\beta \alpha \xi}^{\eta} y^{\lambda} y^{\xi}\right] \\
& K_{(n+\alpha) \beta(n+\gamma)}^{\delta}=-\frac{1}{2} \frac{\partial}{\partial x^{n+\gamma}}\left[R_{\beta \alpha \lambda}^{\delta} y^{\lambda}\right]-\frac{1}{4} R_{\epsilon \gamma \mu}^{\delta} R_{\beta \alpha \nu}^{\epsilon} y^{\mu} y^{v} \\
& K_{(n+\alpha) \beta(n+\gamma)}^{(n+\delta)}=-\frac{\partial}{\partial x^{(n+\gamma)}}\left[\Gamma_{\alpha \beta}^{\delta}-\frac{1}{2} \Gamma_{\mu \lambda}^{\delta} R_{\beta \alpha \nu}^{\lambda} y^{\mu} y^{\nu}\right]-\frac{1}{2}\left[\Gamma_{\gamma \epsilon}^{\delta}-\frac{1}{2} \Gamma_{\mu \lambda}^{\delta} R_{\epsilon \gamma \nu}^{\lambda} y^{\mu} y^{\nu}\right] R_{\beta \alpha \kappa}^{\epsilon} y^{\kappa} \\
& K_{\alpha \beta \gamma}^{\delta}=\frac{\partial}{\partial x^{\beta}}\left[\Gamma_{\alpha \gamma}^{\delta}+\frac{1}{2}\left(R_{\gamma \kappa \mu}^{\delta} \Gamma^{\kappa}{ }_{\nu \alpha}+R_{\alpha \kappa \mu}^{\delta} \Gamma^{\kappa^{\alpha} \gamma}\right) y^{\mu} y^{\nu}\right]-\frac{\partial}{\partial x^{\gamma}}\left[\Gamma_{\alpha \beta}^{\delta}+\frac{1}{2}\left(R_{\beta \kappa \mu}^{\delta} \Gamma^{\kappa}{ }_{v \alpha}+R_{\alpha \kappa \mu}^{\delta} \Gamma_{\nu \beta}^{\kappa}\right) y^{\mu} y^{\nu}\right] \\
& +\left[\Gamma_{\beta \epsilon}^{\delta}+\frac{1}{2}\left(R^{\delta}{ }_{\epsilon \kappa \mu} \Gamma^{\kappa}{ }_{\nu \beta}+R_{\beta \kappa \mu}^{\delta} \Gamma^{\kappa}{ }_{\nu \epsilon}\right) y^{\mu} y^{\nu}\right]\left[\Gamma_{\alpha \gamma}^{\epsilon}+\frac{1}{2}\left(R_{\gamma \kappa \lambda}^{\delta} \Gamma^{\kappa}{ }^{\kappa}{ }_{\xi \alpha}+R^{\delta}{ }_{\alpha \kappa \lambda} \Gamma^{\kappa}{ }_{\xi \gamma}\right) y^{\lambda} y^{\xi}\right]
\end{aligned}
$$

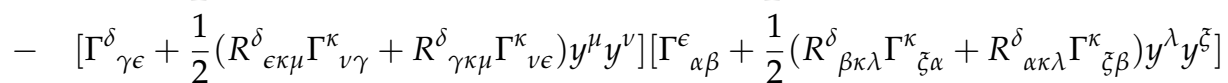

$$
\begin{aligned}
& +\frac{1}{4} R_{\beta \epsilon \epsilon}^{\delta} y^{\xi}\left[\left(R_{\alpha \lambda \gamma}^{\epsilon}+R_{\gamma \lambda \alpha}^{\epsilon}+2 \frac{\partial \Gamma^{\epsilon}{ }_{\alpha \gamma}}{\partial x^{\lambda}}\right) y^{\lambda}+\Gamma^{\epsilon}{ }_{\nu \kappa}\left(R_{\gamma \mu \eta}^{\kappa} \Gamma_{\lambda \alpha}^{\eta}+R_{\alpha \mu \eta}^{\kappa} \Gamma_{\lambda \gamma}^{\eta}\right) y^{\lambda} y^{\mu} y^{\nu}\right] \\
& -\frac{1}{4} R_{\gamma \epsilon \xi}^{\delta} y^{\xi}\left[\left(R_{\alpha \lambda \beta}^{\epsilon}+R_{\beta \lambda \alpha}^{\epsilon}+2 \frac{\partial \beta_{\alpha \beta}^{\epsilon}}{\partial x^{\lambda}}\right) y^{\lambda}+\beta_{\nu \kappa}^{\epsilon}\left(R_{\beta \mu \eta}^{\kappa} \beta_{\lambda \alpha}^{\eta}+R_{\alpha \mu \eta}^{\kappa} \beta_{\lambda \beta}^{\eta}\right) y^{\lambda} y^{\mu} y^{\nu}\right] \\
& K_{\alpha \beta \gamma}^{(n+\delta)}=\frac{1}{2} \frac{\partial}{\partial x^{\beta}}\left[\left(R_{\alpha \lambda \gamma}^{\delta}+R_{\gamma \lambda \alpha}^{\delta}+2 \frac{\partial \Gamma_{\alpha \gamma}^{\delta}}{\partial x^{\lambda}}\right) y^{\lambda}+\Gamma^{\delta}{ }_{\nu \kappa}\left(R^{\kappa}{ }_{\gamma \mu \eta} \Gamma_{\lambda \alpha}^{\eta}+R_{\alpha \mu \eta}^{\kappa} \Gamma_{\lambda \gamma}^{\eta}\right) y^{\lambda} y^{\mu} y^{v}\right] \\
& -\frac{1}{2} \frac{\partial}{\partial x^{\gamma}}\left[\left(R_{\alpha \lambda \beta}^{\delta}+R_{\beta \lambda \alpha}^{\delta}+2 \frac{\partial \Gamma_{\alpha \beta}^{\delta}}{\partial x^{\lambda}}\right) y^{\lambda}+\Gamma_{\nu \kappa}^{\delta}\left(R_{\beta \mu \eta}^{\kappa} \Gamma_{\lambda \alpha}^{\eta}+R_{\alpha \mu \eta}^{\kappa} \Gamma_{\lambda \beta}^{\eta}\right) y^{\lambda} y^{\mu} y^{\nu}\right] \\
& +\frac{1}{2}\left[\left(R_{\beta \lambda \epsilon}^{\delta}+R_{\epsilon \lambda \beta}^{\delta}+2 \frac{\partial \Gamma_{\beta \epsilon}^{\delta}}{\partial x^{\lambda}}\right) y^{\lambda}+\Gamma_{\nu \kappa}^{\delta}\left(R_{\epsilon \mu \eta}^{\kappa} \Gamma_{\lambda \beta}^{\eta}+R_{\beta \mu \eta}^{\kappa} \Gamma_{\lambda \epsilon}^{\eta}\right) y^{\lambda} y^{\mu} y^{\nu}\right] . \\
& {\left[\Gamma_{\alpha \gamma}^{\epsilon}+\frac{1}{2}\left(R_{\gamma \kappa \xi}^{\epsilon} \Gamma^{\kappa}{ }_{\rho \alpha}+R_{\alpha \kappa \xi}^{\epsilon} \Gamma^{\kappa}{ }^{\kappa}\right) y^{\rho} y^{\xi}\right]} \\
& -\frac{1}{2}\left[\left(R_{\gamma \lambda \epsilon}^{\delta}+R_{\epsilon \lambda \gamma}^{\delta}+2 \frac{\partial \Gamma_{\gamma \epsilon}^{\delta}}{\partial x^{\lambda}}\right) y^{\lambda}+\Gamma_{\nu \kappa}^{\delta}\left(R_{\epsilon \mu \eta}^{\kappa} \Gamma_{\lambda \gamma}^{\eta}+R_{\gamma \mu \eta}^{\kappa} \Gamma_{\lambda \epsilon}^{\eta}\right) y^{\lambda} y^{\mu} y^{\nu}\right] \\
& {\left[\Gamma_{\alpha \beta}^{\epsilon}+\frac{1}{2}\left(R_{\beta \kappa \xi}^{\epsilon} \Gamma^{\kappa}{ }^{\kappa}{ }_{\rho \alpha}+R_{\alpha \kappa \xi}^{\epsilon} \Gamma^{\kappa}{ }_{\rho \beta}\right) y^{\rho} y^{\xi}\right]} \\
& +\frac{1}{2}\left[\left(R_{\alpha \lambda \gamma}^{\epsilon}+R_{\gamma \lambda \alpha}^{\epsilon}+2 \frac{\partial \Gamma_{\alpha \gamma}^{\epsilon}}{\partial x^{\lambda}}\right) y^{\lambda}+\Gamma^{\epsilon}{ }_{\nu \kappa}\left(R^{\kappa}{ }_{\gamma \mu \eta} \Gamma_{\lambda \alpha}^{\eta}+R^{\kappa}{ }_{\alpha \mu \eta} \Gamma_{\lambda \gamma}^{\eta}{ }_{\lambda \gamma}\right) y^{\lambda} y^{\mu} y^{\nu}\right]\left[\Gamma_{\epsilon \beta}^{\delta}-\frac{1}{2} R^{\kappa}{ }_{\beta \epsilon \zeta} \Gamma^{\Gamma^{\delta}}{ }_{\rho \kappa} y^{\rho} y^{\xi}\right] \\
& -\frac{1}{2}\left[\left(R_{\alpha \lambda \beta}^{\epsilon}+R_{\beta \lambda \alpha}^{\epsilon}+2 \frac{\partial \Gamma_{\alpha \beta}^{\epsilon}}{\partial x^{\lambda}}\right) y^{\lambda}+\Gamma_{\nu \kappa}^{\epsilon}\left(R_{\beta \mu \eta}^{\kappa} \Gamma_{\lambda \alpha}^{\eta}+R_{\alpha \mu \eta}^{\kappa} \Gamma_{\lambda \beta}^{\eta}\right) y^{\lambda} y^{\mu} y^{\nu}\right]\left[\Gamma_{\epsilon \gamma}^{\delta}-\frac{1}{2} R^{\kappa}{ }_{\gamma \epsilon \zeta} \Gamma^{\delta}{ }_{\rho \kappa} y^{\rho} y^{\xi}\right]
\end{aligned}
$$




$$
\begin{aligned}
& K_{\alpha \beta(n+\gamma)}^{\delta}=\frac{1}{2} \frac{\partial}{\partial x^{\beta}}\left[R_{\alpha \gamma \lambda}^{\delta} y^{\lambda}\right]-\frac{\partial}{\partial x^{(n+\gamma)}}\left[\Gamma_{\alpha \beta}^{\delta}-\frac{1}{2}\left(R_{\beta \kappa \mu}^{\delta} \Gamma_{\lambda \alpha}^{\kappa}+R_{\alpha \kappa \mu}^{\delta} \Gamma_{\lambda \beta}^{\kappa}\right) y^{\lambda} y^{\mu}\right] \\
& +\frac{1}{2} R_{\beta \epsilon \nu}^{\delta} y^{\nu}\left[\Gamma_{\gamma \alpha}^{\epsilon}-\frac{1}{2} R_{\alpha \gamma \lambda}^{\kappa} \Gamma_{\mu \kappa}^{\epsilon} y^{\lambda} y^{\mu}\right] \\
& +\frac{1}{2} R_{\alpha \gamma \nu}^{\epsilon} y^{v}\left[\Gamma_{\beta \epsilon}^{\delta}+\frac{1}{2}\left(R_{\epsilon \kappa \mu}^{\delta} \Gamma_{\lambda \beta}^{\kappa}+R_{\beta \kappa \mu}^{\delta} \Gamma_{\lambda \epsilon}^{\kappa}\right) y^{\lambda} y^{\mu}\right] \\
& -\frac{1}{2} R_{\epsilon \gamma \nu}^{\delta} y^{\nu}\left[\Gamma_{\alpha \beta}^{\epsilon}+\frac{1}{2}\left(R_{\beta \kappa \mu}^{\epsilon} \Gamma_{\lambda \alpha}^{\kappa}+R_{\alpha \kappa \mu}^{\epsilon} \Gamma_{\lambda \beta}^{\kappa}\right) y^{\lambda} y^{\mu}\right] \\
& K_{\alpha \beta(n+\gamma)}^{(n+\delta)}=\frac{\partial}{\partial x^{\beta}}\left[\Gamma_{\alpha \gamma}^{\delta}-\frac{1}{2} R_{\alpha \gamma \lambda}^{\kappa} \Gamma_{\mu \kappa}^{\delta} y^{\lambda} y^{\mu}\right] \\
& -\frac{1}{2} \frac{\partial}{\partial x^{(n+\gamma)}}\left[\left(R_{\alpha \lambda \beta}^{\delta}+R_{\beta \lambda \alpha}^{\delta}+2 \frac{\partial \Gamma_{\alpha \beta}^{\delta}}{\partial x^{\lambda}}\right) y^{\lambda}+\Gamma_{\nu \kappa}^{\delta}\left(R_{\beta \mu \eta}^{\kappa} \Gamma_{\lambda \alpha}^{\eta}+R_{\alpha \mu \eta}^{\kappa} \Gamma_{\lambda \beta}^{\eta}\right) y^{\lambda} y^{\mu} y^{v}\right] \\
& +\frac{1}{2} R_{\alpha \gamma \rho}^{\epsilon} y^{\rho}\left[\left(R_{\beta \lambda \epsilon}^{\delta}+R_{\epsilon \lambda \beta}^{\delta}+2 \frac{\partial \Gamma_{\beta \epsilon}^{\delta}}{\partial x^{\lambda}}\right) y^{\lambda}+\Gamma_{\nu \kappa}^{\delta}\left(R_{\epsilon \mu \eta}^{\kappa} \Gamma_{\lambda \beta}^{\eta}+R_{\beta \mu \eta}^{\kappa} \Gamma_{\lambda \epsilon}^{\eta}\right) y^{\lambda} y^{\mu} y^{\nu}\right] \\
& +\left[\Gamma_{\gamma \alpha}^{\epsilon}-\frac{1}{2} R^{\kappa}{ }_{\alpha \gamma \lambda} \Gamma_{\mu \kappa}^{\epsilon} y^{\lambda} y^{\mu}\right]\left[\Gamma_{\epsilon \beta}^{\delta}-\frac{1}{2} R_{\beta \epsilon \epsilon^{\kappa}}^{\kappa} \Gamma_{\rho \kappa}^{\delta} y^{\rho} y^{\xi}\right] \\
& -\left[\Gamma_{\alpha \beta}^{\epsilon}+\frac{1}{2}\left(R_{\beta \kappa v}^{\epsilon} \Gamma_{\rho \alpha}^{\kappa}+R_{\alpha \kappa \nu}^{\epsilon} \Gamma_{\rho \beta}^{\kappa}\right) y^{\rho} y^{\nu}\right]\left[\Gamma_{\epsilon \gamma}^{\delta}-\frac{1}{2} R^{\kappa}{ }_{\epsilon \gamma \xi} \Gamma^{\delta}{ }_{\mu \kappa} y^{\mu} y^{\xi}\right] \\
& K_{\alpha(n+\beta) \gamma}^{\delta}=\frac{\partial}{\partial x^{(n+\beta)}}\left[\Gamma_{\alpha \gamma}^{\delta}+\frac{1}{2}\left(R_{\gamma \kappa \mu}^{\delta} \Gamma_{\lambda \alpha}^{\kappa}+R_{\alpha \kappa \mu}^{\delta} \Gamma_{\lambda \gamma}^{\kappa}\right) y^{\lambda} y^{\mu}\right]-\frac{1}{2} \frac{\partial}{\partial x^{\gamma}}\left[R_{\alpha \beta \lambda}^{\delta} y^{\lambda}\right] \\
& +\frac{1}{2} R_{\epsilon \beta \nu}^{\delta} y^{v}\left[\Gamma_{\gamma \alpha}^{\epsilon}+\frac{1}{2}\left(R_{\gamma \kappa \mu}^{\epsilon} \Gamma_{\lambda \alpha}^{\kappa}+R_{\alpha \kappa \mu}^{\epsilon} \Gamma_{\lambda \gamma}^{\kappa}\right) y^{\lambda} y^{\mu}\right] \\
& -\frac{1}{2} R_{\alpha \beta \nu}^{\epsilon} y^{\nu}\left[\Gamma_{\gamma \epsilon}^{\delta}+\frac{1}{2}\left(R_{\epsilon \kappa \mu}^{\delta} \Gamma_{\lambda \gamma}^{\kappa}+R_{\gamma \kappa \mu}^{\delta} \Gamma_{\lambda \epsilon}^{\kappa}\right) y^{\lambda} y^{\mu}\right] \\
& -\frac{1}{2} R_{\gamma \epsilon \nu}^{\delta} y^{\nu}\left[\Gamma_{\beta \alpha}^{\epsilon}-\frac{1}{2} R_{\alpha \beta \lambda}^{\kappa} \Gamma_{\mu \kappa}^{\epsilon} y^{\lambda} y^{\mu}\right] \\
& K_{\alpha(n+\beta) \gamma}^{(n+\delta)}=\frac{1}{2} \frac{\partial}{\partial x^{(n+\beta)}}\left[\left(R_{\alpha \lambda \gamma}^{\delta}+R_{\gamma \lambda \alpha}^{\delta}+2 \frac{\partial \Gamma_{\alpha \gamma}^{\delta}}{\partial x^{\lambda}}\right) y^{\lambda}+\Gamma_{\nu \mathcal{K}}^{\delta}\left(R_{\gamma \mu \eta}^{\kappa} \Gamma_{\lambda \alpha}^{\eta}+R_{\alpha \mu \eta}^{\kappa} \Gamma_{\lambda \gamma}^{\eta}\right) y^{\lambda} y^{\mu} y^{\nu}\right] \\
& -\frac{\partial}{\partial x^{\gamma}}\left[\Gamma_{\alpha \beta}^{\delta}-\frac{1}{2} R_{\alpha \beta \lambda}^{\kappa} \Gamma_{\mu \kappa}^{\delta} y^{\lambda} y^{\mu}\right] \\
& -\frac{1}{2} R_{\alpha \beta \rho}^{\epsilon} y^{\rho}\left[\left(R_{\gamma \lambda \epsilon}^{\delta}+R_{\epsilon \lambda \gamma}^{\delta}+2 \frac{\partial \Gamma_{\gamma \epsilon}^{\delta}}{\partial x^{\lambda}}\right) y^{\lambda}+\Gamma^{\delta}{ }_{\nu \mathcal{K}}\left(R_{\epsilon \mu \eta}^{\kappa} \Gamma_{\lambda \gamma}^{\eta}+R_{\gamma \mu \eta}^{\kappa} \Gamma_{\lambda \epsilon}^{\eta}\right) y^{\lambda} y^{\mu} y^{\nu}\right] \\
& \text { - }\left[\Gamma_{\beta \alpha}^{\epsilon}-\frac{1}{2} R_{\alpha \beta \lambda}^{\kappa} \Gamma_{\mu \kappa}^{\epsilon} y^{\lambda} y^{\mu}\right]\left[\Gamma_{\epsilon \gamma}^{\delta}-\frac{1}{2} R_{\gamma \epsilon \xi}^{\kappa} \Gamma_{\rho \kappa}^{\delta} y^{\rho} y^{\xi}\right] \\
& +\left[\Gamma_{\alpha \gamma}^{\epsilon}+\frac{1}{2}\left(R_{\gamma \kappa \nu}^{\epsilon} \Gamma_{\rho \alpha}^{\kappa}+R_{\alpha \kappa \nu}^{\epsilon} \Gamma_{\rho \gamma}^{\kappa}\right) y^{\rho} y^{v}\right]\left[\Gamma_{\epsilon \beta}^{\delta}-\frac{1}{2} R^{\kappa}{ }_{\epsilon \beta \xi^{\xi}} \Gamma_{\mu \kappa}^{\delta} y^{\mu} y^{\xi}\right] \\
& K_{\alpha(n+\beta)(n+\gamma)}^{\delta}=\frac{1}{2} \frac{\partial}{\partial x^{(n+\beta)}}\left[R_{\gamma \alpha \lambda}^{\delta} y^{\lambda}\right]-\frac{1}{2} \frac{\partial}{\partial x^{(n+\gamma)}}\left[R_{\beta \alpha \lambda}^{\delta} y^{\lambda}\right]+\frac{1}{4} R_{\epsilon \beta \nu}^{\delta} R_{\alpha \gamma \mu}^{\epsilon} y^{\mu} y^{v}-\frac{1}{4} R_{\epsilon \gamma \nu}^{\delta} R_{\alpha \beta \mu}^{\epsilon} y^{\mu} y^{v}
\end{aligned}
$$




$$
\begin{aligned}
K_{\alpha(n+\beta) \gamma}^{(n+\delta)} & =\frac{\partial}{\partial x^{(n+\beta)}}\left[\Gamma_{\alpha \gamma}^{\delta}-\frac{1}{2} R_{\alpha \gamma \lambda}^{\kappa} \Gamma_{\mu \kappa}^{\delta} y^{\lambda} y^{\mu}\right]-\frac{\partial}{\partial x^{(n+\gamma)}}\left[\Gamma_{\alpha \beta}^{\delta}-\frac{1}{2} R^{\kappa}{ }_{\alpha \beta \lambda} \Gamma_{\mu \kappa}^{\delta} y^{\lambda} y^{\mu}\right] \\
& +\left[\Gamma_{\alpha \gamma}^{\epsilon}+\frac{1}{2}\left(R_{\gamma \kappa \nu}^{\epsilon} \Gamma_{\rho \alpha}^{\kappa}+R_{\alpha \kappa \nu}^{\epsilon} \Gamma_{\rho \gamma}^{\kappa}\right) y^{\rho} y^{\nu}\right]\left[\Gamma_{\epsilon \beta}^{\delta}-\frac{1}{2} R_{\epsilon \beta \xi^{\kappa}} \Gamma_{\mu \kappa}^{\delta} y^{\mu} y^{\xi}\right] \\
& -\left[\Gamma_{\alpha \beta}^{\epsilon}+\frac{1}{2}\left(R_{\beta \kappa \nu}^{\epsilon} \Gamma_{\rho \alpha}^{\kappa}+R_{\alpha \kappa \nu}^{\epsilon} \Gamma_{\rho \beta}^{\kappa}\right) y^{\rho} y^{\nu}\right]\left[\Gamma^{\delta}{ }_{\epsilon \gamma}-\frac{1}{2} R^{\kappa}{ }_{\epsilon \gamma \xi^{\xi}} \Gamma^{\delta}{ }_{\mu \kappa} y^{\mu} y^{\xi}\right]
\end{aligned}
$$

where $\Gamma_{\beta \gamma}^{\alpha} R_{\alpha \beta \gamma}^{\delta}$ are the Christoffel symbols and Riemann curvature tensor of the $n$-dimensional base manifold $(M, g)$, respectively, and, inkeeping with our previous convention for Section 3 , the greek indices $\{\alpha, \beta, \ldots\}=\{1,2, \cdots n\}$ and the latin indices $\{a, b, \ldots\}=\{1,2, \cdots 2 n\}$.

It should be noted that if the base manifold is flat, i.e., in an appropriate coordinate system $R_{\alpha \beta \gamma}^{\delta}=0$ and $\Gamma_{\beta \gamma}^{\alpha}=0$ then $\bar{\Gamma}^{i}{ }_{j k}=0$ and $K_{j h k}^{i}=0$. Therefore, a flat base manifold induces a flat Sasaki tangent bundle. In this case the "geodesics" of the flat tangent space are all trivially produced by lifting the "geodesics" of the base manifold.

\section{References}

1. Farnes, J.S. A unifying theory of dark energy and dark matter: Negative masses and matter creation within a modified $\Lambda$ CDM framework. Astron. Astrophys. 2018, 620, A92. [CrossRef]

2. Nadler, E.O.; Wechsler, R.H.; Bechtol, K.; Mao, Y.Y.; Green, G.; Drlica-Wagner, A.; McNanna, M.; Mau, S.; Pace, A.B.; Simon, J.D.; et al. Milky Way Satellite Census. II. Galaxy-Halo Connection Constraints Including the Impact of the Large Magellanic Cloud. Astrophys. J. 2020, 893, 48. [CrossRef]

3. Ren, X.; Zhao, L.; Abdukerim, A.; Chen, X.; Chen, Y.; Cui, X.; Fang, D.; Fu, C.; Giboni, K.; Giuliani, F.; et al. Constraining Dark Matter Models with a Light Mediator at the PandaX-II Experiment. Phys. Rev. Lett. 2018, 121, 021304. [CrossRef] [PubMed]

4. Dror, J.A.; Elor, G.; McGehee, R. Directly Detecting Signals from Absorption of Fermionic Dark Matter. Phys. Rev. Lett. 2020, 124, 181301. [CrossRef]

5. Hsueh, J.W.; Enzi, W.; Vegetti, S.; Auger, M.W.; Fassnacht, C.D.; Despali, G.; Koopmans, L.V.; McKean, J.P. SHARP-VII. New constraints on the dark matter free-streaming properties and substructure abundance from gravitationally lensed quasars. Mon. Not. R. Astron. Soc. 2020, 492, 3047-3059. [CrossRef]

6. Riess, A.G.; Filippenko, A.V.; Challis, P.; Clocchiatti, A.; Diercks, A.; Garnavich, P.M.; Gilliland; R.L.; Hogan, C.J.; Jha, S.; Kirshner, R.P.; et al. Observational evidence from supernovae for an accelerating universe and a cosmological constant. Astron. J. 1998, 116, 1009-1038. [CrossRef]

7. Rigault, M.; Brinnel, V.; Aldering, G.; Antilogus, P.; Aragon, C.; Bailey, S.; Baltay, C.; Barbary, K.; Bongard, S.; Boone, K.; et al. Strong Dependence of Type Ia Supernova Standardization on the Local Specific Star Formation Rate. arXiv 2018, arXiv:1806.03849.

8. Martinelli, M.; Tutusaus, I. CMB tensions with low-redshift H0 and S8 measurements: Impact of a redshift-dependent type-Ia supernovae intrinsic luminosity. Symmetry 2019, 11, 986. [CrossRef]

9. Di Valentino, E.; Gariazzo, S.; Mena, O.; Vagnozzi, S. Soundness of Dark Energy Properties. J. Cosmol. Astropart. Phys. 2020. [CrossRef]

10. Dhawan, S.; Jha S.W.; Leibundgut, B. Measuring the Hubble constant with Type Ia supernovae as near-infrared standard candles. Astron. Astrophys. 2018, 609, A72. [CrossRef]

11. Leibundgut, B.; Blondin, S. Evidence for dark energy from Type Ia supernovae. Nucl. Phys. B Proc. Suppl. 2005, 138, 10-15. [CrossRef]

12. Krauss, L. M.; Jones-Smith, K.; Huterer, D. Dark energy, a cosmological constant, and type Ia supernovae. New J. Phys. 2007, 9, 141.

13. Perlmutter, S. Supernovae, Dark Energy, and the Accelerating Universe. Phys. Today 2003, 56, 53. [CrossRef]

14. Riess, A.G. Type Ia supernova discoveries at $\mathrm{z}>1$ from the Hubble Space Telescope: Evidence for past deceleration and constraints on dark energy evolution. Astrophys. J. 2004, 607, 665-687. [CrossRef]

15. Abbott, T.M.C.; Allam, S.; Andersen, P.; Angus, C.; Asorey, J.; Avelino, A.; Avila, S.; Bassett, B.A.; Bechtol, K.; Bernstein, G.M.; et al. First Cosmology Results using Type Ia Supernovae from the Dark Energy Survey: Constraints on Cosmological Parameters. Astrophys. J. Lett. 2019, 872, L30. [CrossRef] 
16. Kang, Y.; Lee, Y.W.; Kim, Y.L.; Chung, C.; Ree, C.H. Early-type Host Galaxies of Type Ia Supernovae. II. Evidence for Luminosity Evolution in Supernova Cosmology. arXiv 2019, arXiv:1912.04903.

17. Nielsen, J.T.; Guffanti, A.; Sarkar, S. Marginal evidence for cosmic acceleration from Type Ia supernovae. Sci. Rep. 2016, 6, 35596. [CrossRef]

18. Di Valentino, E.; Melchiorri, A.; Linder, E.V.; Silk, J. Constraining dark energy dynamics in extended parameter space. Phys. Rev. D. 2017, 96, 023523. [CrossRef]

19. Leibundgut. B.; Sullivan, M. Type Ia supernova cosmology. Space Sci. Rev. 2018, 214, 57. [CrossRef]

20. Perlmutter, S.; Aldering, G.; Goldhaber, G.; Knop, R.A.; Nugent, P.; Castro, P.G.; Deustua, S.; Fabbro, S.; Goobar, A.; Groom, D.E.; et al. Measurements of $\Omega$ and $\Lambda$ from 42 high-redshift supernovae. Astrophys. J. 1999, 517, 565. [CrossRef]

21. Velten, H.; Gomes, S.; Busti, V.C. Gauging the cosmic acceleration with recent type Ia supernovae data sets. Phys. Rev. D. 2018, 97, 083516. [CrossRef]

22. Hoscheit, B.L.; Barger, A.J. The KBC Void: Consistency with Supernovae Type Ia and the Kinematic SZ Effect in a $\Lambda$ LTB Model. Astrophys. J. 2018, 854, 46. [CrossRef]

23. Gariazzo, S.; Escudero, M.; Diamanti, R.; Mena, O. Cosmological searches for a noncold dark matter component. Phys. Rev. D 2017, 96, 043501. [CrossRef]

24. Di Valentino, E.; Melchiorri, A.; Mena, O.; Vagnozzi, S. Nonminimal dark sector physics and cosmological tensions. Phys. Rev. D 2020, 101, 063502. [CrossRef]

25. Collett, T.E.; Oldham, L.J.; Smith, R.J.; Auger, M.W.; Westfall, K.B.; Bacon, D.; Nichol, R.C.; Masters, K.L.; Koyama, K.; van den Bosch, R. A Precise Extragalactic Test of General Relativity. Science 2018, 360, 1342-1346. [CrossRef]

26. Hartle, J.B. Gravity: An Introduction to Einstein's General Relativity; Addison-Wesley: Boston, MA, USA, 2003.

27. Borowiec, A.; Godłowski, W.; Szydłowski, M. Dark Matter and Dark Energy as Effects of Modified Gravity. Int. J. Geom. Methods Mod. Phys. 2007, 4, 183-196. [CrossRef]

28. Persic, M.; Salucci, P.; Stel, F. The Universal Rotation Curve of Spiral Galaxies: I. the Dark Matter Connection. Mon. Not. R. Astron. Soc. 1995, 283, 27-47.

29. Harko, T.; Lobo, F. Geodesic deviation, Raychaudhuri equation, and tidal forces in modified gravity with an arbitrary curvature-matter coupling. Phys. Rev. D. 2012, 86, 124034. [CrossRef]

30. Harko, T.; Lobo, F. Generalized Curvature-Matter Couplings in Modified Gravity. Galaxies 2014, 2, 410-465. [CrossRef]

31. Sasaki, S. On the differential geometry of tangent bundles of Riemannian manifolds. Tohoku Math. J. 1958, 10, 338-354. [CrossRef]

32. Chamseddine, A.H.; Mukhanov, V. Resolving Cosmological Singularities. J. Cosmol. Astropart. Phys. 2017, 2017, 009. [CrossRef]

33. Ball, P. Dark matter highlights extra dimensions. Nature 2005. [CrossRef]

34. Kahil, M.; Harko, T. Is dark matter an extra-dimensional effect? Mod. Phys. Lett. A 2011, 24, 667-682. [CrossRef]

35. Coimbra Araújo, C.H.; da Rocha, R. Gravity with Extra Dimensions and Dark Matter Interpretation: A Straightforward Approach. ISRN High Energy Phys. 2013, 2013, 713508. [CrossRef]

36. Tanabashi, M. Particle Data Group. Phys. Rev. D 2018, 98, 030001. [CrossRef]

37. Miller, K.S. On the Inverse of the Sum of Matrices. Math. Mag. 1981, 54, 67-72. [CrossRef]

38. Capozziello, S.; Cardone, V.F.; Troisi, A. Dark Energy and Dark Matter as Curvature Effects? J. Cosmol. Astropart. Phys. 2006, 2006. [CrossRef]

39. Raychaudhuri, A. Relativistic Cosmology. Phys. Rev. 1955, 98, 1123. [CrossRef]

40. Hawking, S.; Ellis, G. The Large Scale Structure of Space-Time; Cambridge Monographs on Mathematical Physics; Cambridge University Press: Cambridge, UK, 1973.

41. Kar, S.; Sengupta, S. The Raychaudhuri equations: A brief review. Pramana J. Phys. 2007, 69, 49-76. [CrossRef]

42. Stavrinos, P. C.; Alexiou, M. Raychaudhuri equation in the Finsler-Randers space-time and generalized scalar-tensor theories. Int. J. Geom. Methods Mod. Phys. 2018, 15, 1850039. [CrossRef]

43. Moreshi,O.; Boero, E.; Gallo E.; Gesser, F. Dark matter description by non-conventional energy-momentum tensor. In AIP Conference Proceedings; American Institute of Physics: College Park, MD, USA, 2015; Volume 1647, pp. 35-43.

44. Peebles, P.J.E. Fluid Dark Matter. Astrophys. J. 2000, 534, L127. [CrossRef] [PubMed] 
45. Arbey, A. Dark Fluid: A complex scalar field to unify dark energy and dark matter. Phys. Rev. D 2006, 74, 043516. [CrossRef]

46. Kuzmichev, V.; Kuzmichev, V. Two-component perfect fluid in FRW universe. Acta Phys. Pol. Ser. B 2012, 43, 1899-1910. [CrossRef]

47. Alvarenga, F.; Fracalossi, R.; Freitas, R.C.; Gonçalves, S. Classical and quantum cosmology with two perfect fluids: Stiff matter and radiation. Gen. Relativ. Gravit. 2016, 49, 136. [CrossRef]

48. Ferrando, J.J.; Morales, J.A.; Portilla, M. Two-perfect fluid interpretation of an energy tensor. Gen. Relativ. Gravit. 1990, 22, 1021-1032. [CrossRef]

49. Oliveira, S.R. Model of two perfect fluids for an anisotropic and homogeneous universe. Phys. Rev. D 1989, 40, 3976. [CrossRef]

50. Sotiriou, T.P.; Faraoni, V. F (R) Theor. Gravity. Rev. Mod. Phys. 2010, 82, 451-497. [CrossRef]

51. De Felice, A.; Tsujikawa, S. F (R) Theories. Living Rev. Relativ. 2010, 13, 3. [CrossRef]

52. Capozziello, S.; De Laurentis, M.; Francaviglia, M.; Mercadante, S. From Dark Energy \& Dark Matter to Dark Metric. Found. Phys. 2009, 39, 1161-1176.

53. Nojiri, S.; Odintsov, S.D. Dark energy, inflation and dark matter from modified F(R) gravity. arXiv 2008, arXiv:0807.0685.

54. Tupper, B.O.J. Conformally Ricci-flat viscous fluids. J. Math. Phys. 1990, 31, 1704. [CrossRef]

55. Hansraj, S.; Govinder, K.; Mewalal, N. Conformal Mappings in Relativistic Astrophysics. J. Appl. Math. 2013, 2013, 196385. [CrossRef]

56. de Felice, F.; Clarke, C.J.S. Relativity on Curved Manifolds; Cambridge Monographs on Mathematical Physics; Cambridge University Press: Cambridge, UK, 1990.

57. Carroll, S.M. Spacetime and Geometry: An Introduction to General Relativity; Addison-Wesley: Boston, MA, USA, 2004.

58. Liddle, A.; Lyth, D. Cosmological Inflation and Large-Scale Structure; Cambridge University Press: Cambridge, UK, 2000.

59. Rund, H. The Differential Geometry of Finsler Spaces; Springer: Berlin/Heidelberg, Germany, 1959.

60. Bejan, C.; Gül, I. Sasaki metric on the tangent bundle of a Weyl manifold. Publ. Inst. Math. 2018, 103, 25-32. [CrossRef]

61. Kowalski, O. Curvature of the Induced Riemannian Metric on the Tangent Bundle of a Riemannian Manifold. J. Reine Angew. Math. 1971, 250, 124-129. 